\title{
REVIEW ARTICLE OPEN \\ Nutraceuticals in prostate cancer therapeutic strategies and their neo-adjuvant use in diverse populations
}

\author{
Dominique Reed ${ }^{1}$, Komal Raina ${ }^{1,2}$ and Rajesh Agarwal ${ }^{1,2}$
}

Prostate cancer (PCa) is the most frequently diagnosed malignancy and second leading cause of cancer mortality in American males. Notably, men of African descent in the United States and Caribbean have the highest PCa mortality rates compared to men with European ancestry. Although current therapeutics are quite potent and effective, disease resistance, progression to metastasis, therapy-associated toxicities and efficacy-related issues in diverse populations develop over time. Thus, non-toxic and efficacious therapeutic strategies are needed to address these major obstacles for the clinical treatment and management of PCa. In this regard, preclinical and population-based efficacy studies have shown the potential of natural non-toxic nutraceuticals as potent anti-PCa agents. Accordingly, the implementation of nutraceutical intervention and genetic testing in diverse populations might aid in the development and design of precision medicine strategies to reduce the burden of chemotherapy-associated toxicities, suppress disease resistance, and treat both localized and advanced PCa. Consequently, additional large-scale and inclusive clinical studies are required to fully assess efficacy and therapeutic limitations of these agents in PCa. This review discusses the most current clinical research on selected nutraceutical agents and their efficacy in the context of clinico-pathological outcomes and disease susceptibility in diverse PCa clinical and epidemiological studies.

npj Precision Oncology (2018)2:15 ; doi:10.1038/s41698-018-0058-x

\section{INTRODUCTION}

Despite recent advances in the treatment strategies used for the clinical management of metastatic prostate cancer (PCa), disease survival remains lower than $30 \%$; notably, PCa is the second leading cause of cancer mortality in American men. ${ }^{1}$ Primary interventions for PCa include surgery, adjuvant chemotherapy, hormonal therapy (i.e., androgen deprivation), and/or radiation for advanced disease. Although these therapeutic options are quite potent and effective, disease resistance, progression to metastasis stage, therapy-associated toxicities and efficacy-related issues in diverse populations have developed over time. Thus, efficacious and non-toxic therapeutic strategies are needed to address these major obstacles for the clinical treatment and management of PCa. Past and ongoing chemoprevention/intervention research has recognized the use of nutraceutical agents as a feasible and non-toxic option, which could protect against tumorigenesis as well as enhance the therapeutic response of pre-existing anticancer treatments. $^{2}$

Precision medicine using a nutraceutical approach is one of the key paradigms for the conceptualization and development of nontoxic therapeutic strategies that act synergistically with existing clinical anti-cancer agents. Precision medicine strategies navigate the intricate interplay between genetic, racial, and socio-economic factors, and involve interventions that compensate for individual variations in pharmacogenomic responses to cancer therapies and major PCa risk factors (i.e., age, family history, race, and genetic susceptibility) shown in Fig. 1. Genetic dispositions are critical for identifying individuals at higher risk for disease development or advanced disease and predicting therapeutic response to certain anti-cancer agents. Genetic variations have also shown protective and negative effects on PCa susceptibility. Thus, the efficacy of nutraceutical interventions is heavily influenced by ethnicity and genetic variations of patients. Overall, the relationship between nutraceutical agents, genetic variations, and other chemotherapeutic agents in different populations are not well understood in chemoprevention. Precision medicine strategies could provide a platform to address efficacy issues in PCa chemoprevention/ intervention utilizing both chemotherapeutic and nutraceutical agents; however, additional clinical population studies are needed to confirm their efficacy against genetic, dietary, and environmental factors associated with PCa.

Precision medicine strategies utilize genomic analysis of $\mathrm{PCa}$ patients to identify and elucidate the pharmacogenomic landscape of patient susceptibility for the implementation of adjunct agents that can enhance the anti-tumor activity of pre-existing chemopreventive agents against advanced and/or resistant disease. This approach can aid in designing more specific treatment regimens for high-risk PCa patients. This review will evaluate the most current clinical research (summarized in Tables 1 and 2) on selected nutraceutical agents (silibinin, grapeseed extract, lycopene, soy isoflavanoids, green/black tea, vitamin E, vitamin D, zinc, and selenium) that may serve as adjunct agents in PCa. The efficacy of these nutraceutical agents will be discussed in the context of their effect on clinico-pathological outcomes (i.e., Gleason score, tumor grade, survival, biochemical recurrence), and disease susceptibility in diverse populations based on clinical and epidemiological data. We will also identify research gaps associated with diverse populations and selected nutraceuticals in PCa.

\footnotetext{
${ }^{1}$ Department of Pharmaceutical Sciences, Skaggs School of Pharmacy and Pharmaceutical Sciences, Aurora, CO, USA and ${ }^{2}$ University of Colorado Cancer Center, University of Colorado Anschutz Medical Campus, Aurora, CO, USA

Correspondence: Rajesh Agarwal (Rajesh.Agarwal@UCDenver.edu)
}

Received: 5 February 2018 Revised: 18 June 2018 Accepted: 21 June 2018

Published online: 25 July 2018 


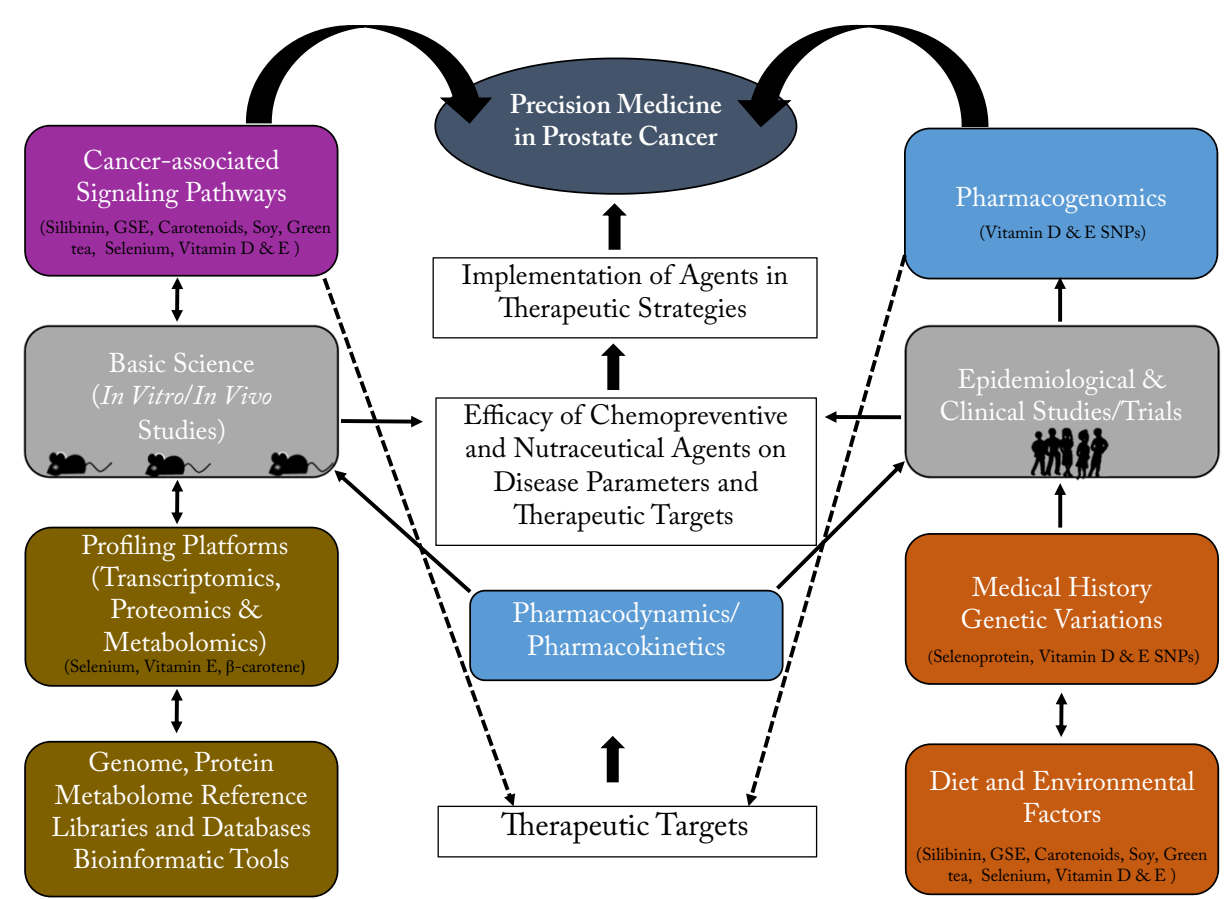

Fig. 1 Nutraceutical efficacy in precision medicine. The schematic above depicts a workflow of experimental designs and assessment parameters required to establish the efficacy of nutraceutical agents. Therapeutic potential of agents must undergo vigorous pharmacodynamics and pharmacokinetics (blue) evaluation of their antioxidant and anti-cancer properties (e.g., anti-proliferation, antigrowth, anti-motility, anti-invasion) in cell models (gray). Targets that regulate altered tumor phenotypes are assessed via cell-focus assays (qRT-PCR, western blot, and immunofluorescence) and high-throughput platforms. Network analyses of targets are performed using omic profiling databases and libraries (brown) to determine gene ontology and identify therapeutic targets in cancer-associated canonical/noncanonical pathways (purple). Therapeutic targets and nutraceutical agents are evaluated in preclinical models and undergo the previous workflow. Next, nutraceutical agents are assessed in epidemiological studies and clinical trials (gray) which can evaluate hereditary, genetic, and environmental factors that range in degree from Phase I ( $\leq 30$ patients, Pharmacodynamics/Pharmacokinetics parameters), Phase II ( $2-3$ treatment groups including standard treatment + new agent, different doses, safety and toxicity assessments, and Pharmacogenomics in humans or animals), Phase III (comparison between new agent and standard treatments, Pharmacogenomics (blue)) (gray), Phase IV (Pharmacogenetic testing and side effects in different populations) to marketing and therapeutic application. After clinical trials have assessed the efficacy of nutraceuticals, these agents can be implemented in current precision medicine clinical therapeutic strategies for patients. The images of the mice and the group of people shown here are created by the authors.

\section{Silibinin}

Over the past two decades, silibinin (flavanolignan from milk thistle "Silybum marianum" seeds) has shown strong anticarcinogenic effects against various types of tumors including $\mathrm{PCa} .{ }^{2,3}$ The significant anti-cancer efficacy of silibinin observed in preclinical animal models of PCa led to its transition into a phase II clinical trial to evaluate its bioavailability in patients diagnosed with localized PCa disease. ${ }^{4}$ Prior to surgery, PCa patients either received $13 \mathrm{~g}$ of silybin-phytosome for $14-31$ days $(n=6)$ or served as untreated control subjects $(n=6)$. High dose of oral silybin-phytosome achieved high plasma concentrations in patients; however, very small amount of silibinin was observed in prostatic tissue due to its short half-life. ${ }^{4}$ Although serum prostate-specific antigen (PSA) levels of patients did not achieve a partial or complete response to silibinin treatment, disease stability was maintained in several patients. Thus, larger clinical studies are still needed to be performed with a more bioavailable form of silibinin to validate its biological efficacy as an effective nutraceutical agent for the clinical management of localized or advanced forms of PCa.

\section{Grape seed extract (GSE)}

GSE is a complex mixture of polyphenols containing procyanidins and their gallate derivatives together known as the proanthocyanidins. ${ }^{5}$ It has shown anti-cancer efficacy against PCa growth and progression in several preclinical models ${ }^{6}$; however, clinical studies have not fully evaluated GSE efficacy in PCa patients.
Interestingly, in a 2011 prospective study "VITamins And Lifestyle (VITAL) $^{\prime 7} \quad(n=1602 ;$ PCa cases; $n=35,239$ total participants), analyzing the biological outcomes of intake of several dietary supplements (for approximately 5-10 years), GSE consumption stood out as the one associated with reduced risk for PCa $(41 \%$ reduction in the risk of mortality among PCa patients relative to non-users). ${ }^{7}$ Importantly, we along with a team of medical oncologists have recently initiated a phase II study of GSE product in asymptomatic or minimally symptomatic non-metastatic PCa patients with rising PSA (NCT03087903), wherein GSE efficacy will be examined in a cohort of PCa survivors who have undergone treatment but show signs of rising PSA after local therapies. Given that current clinical studies have not yet identified molecular signatures modulated by GSE in PCa patients and this agent is a widely consumed as a supplement and food additive, more studies are needed to identify synergistic and/or additive interactions of GSE and its constituents with clinically used antiPCa agents.

\section{Lycopene and other carotenoids}

Lycopene is a powerful carotenoid antioxidant with anti-tumor activity and present in red fruits and vegetables (i.e., tomatoes (tomato-based products), grapefruit, watermelons, and papayas). ${ }^{8}$ Other carotenoids-related compounds such as a-carotene, $\beta$ carotene, $\beta$-cryptoxanthin, xanthophyll carotenoids, lutein, and retinol have also shown anti-tumor activity. Interestingly, $\alpha$ carotene, $\beta$-carotene, and $\beta$-cryptoxanthin can be converted into 


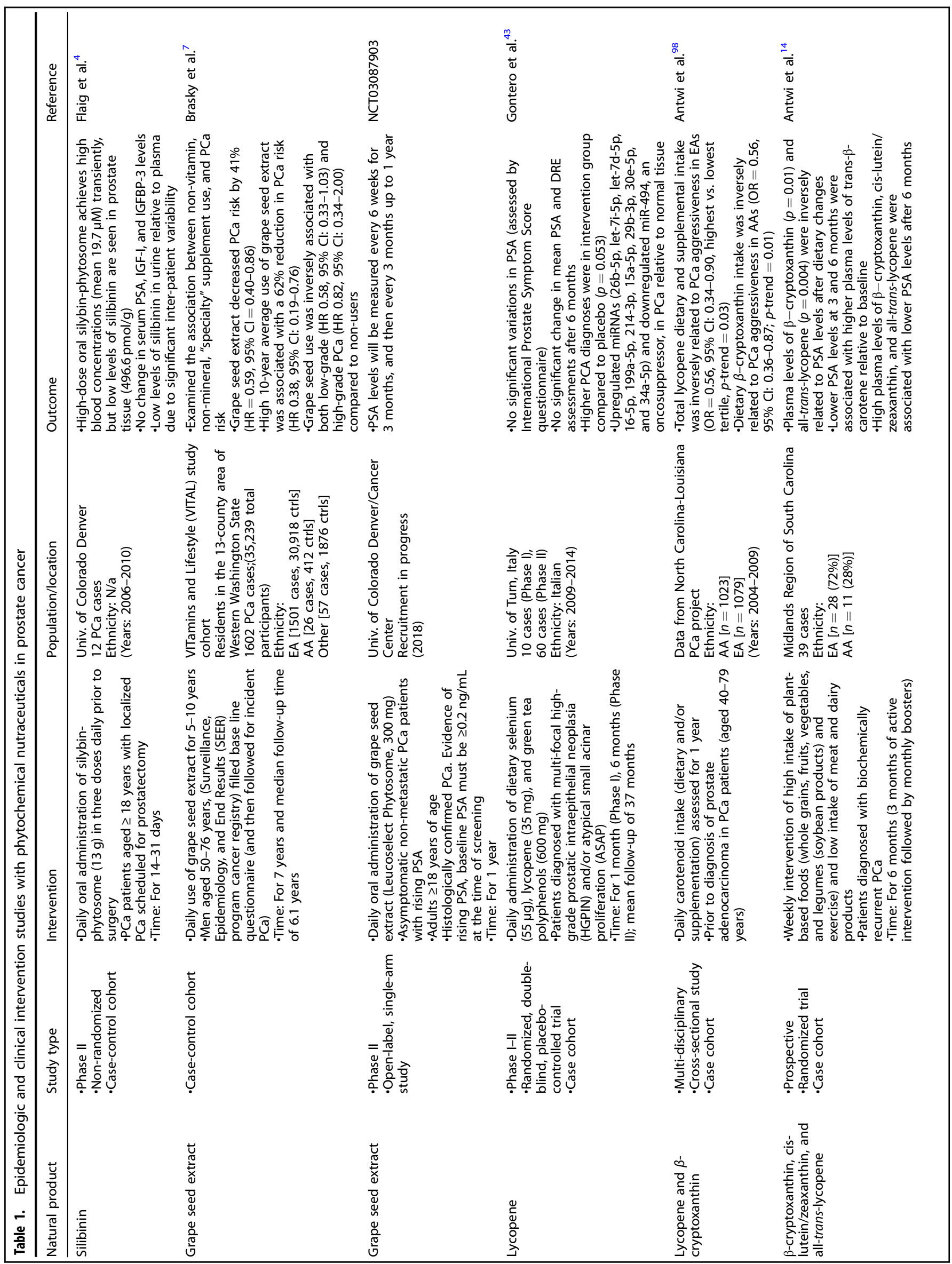




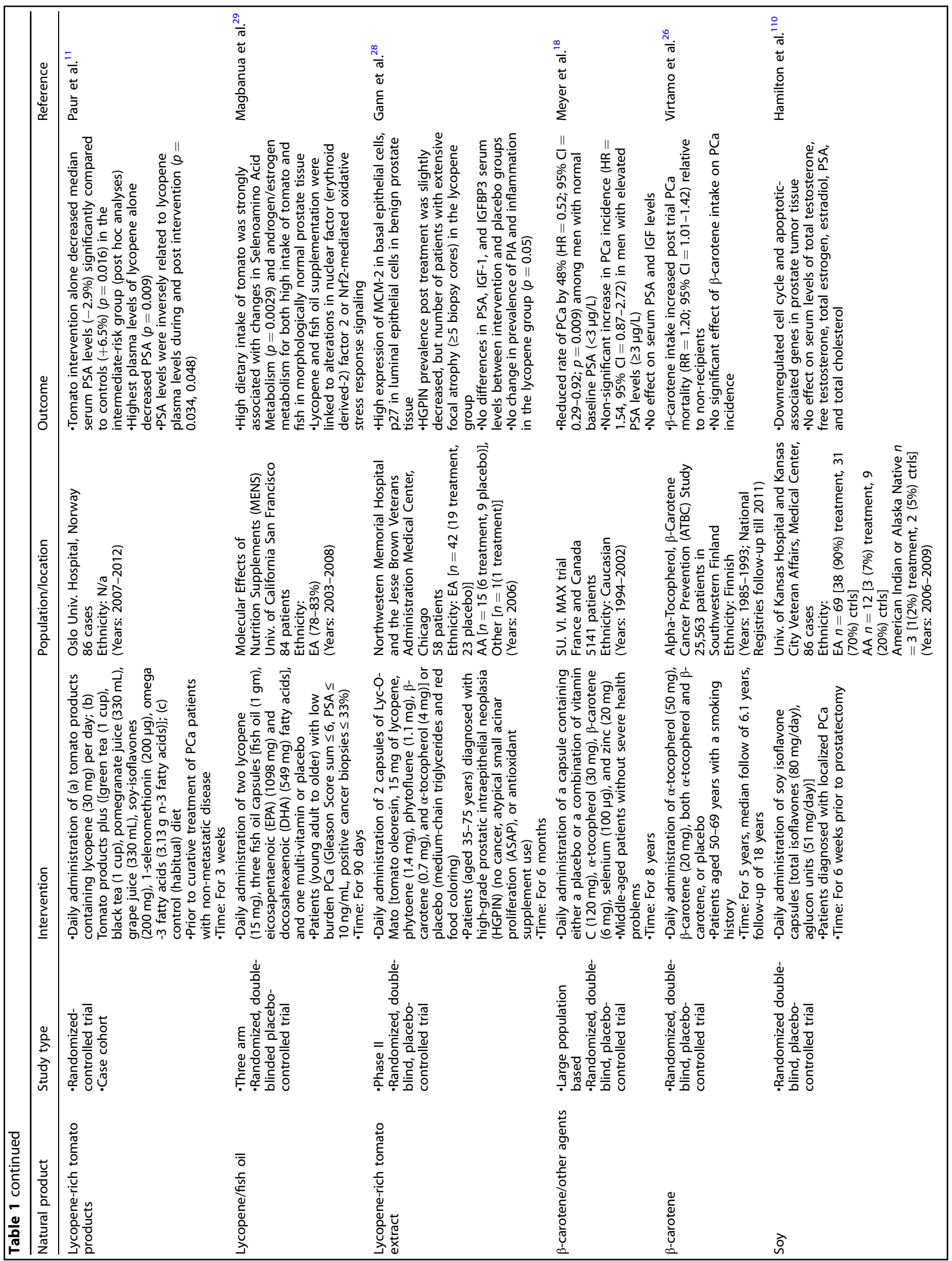




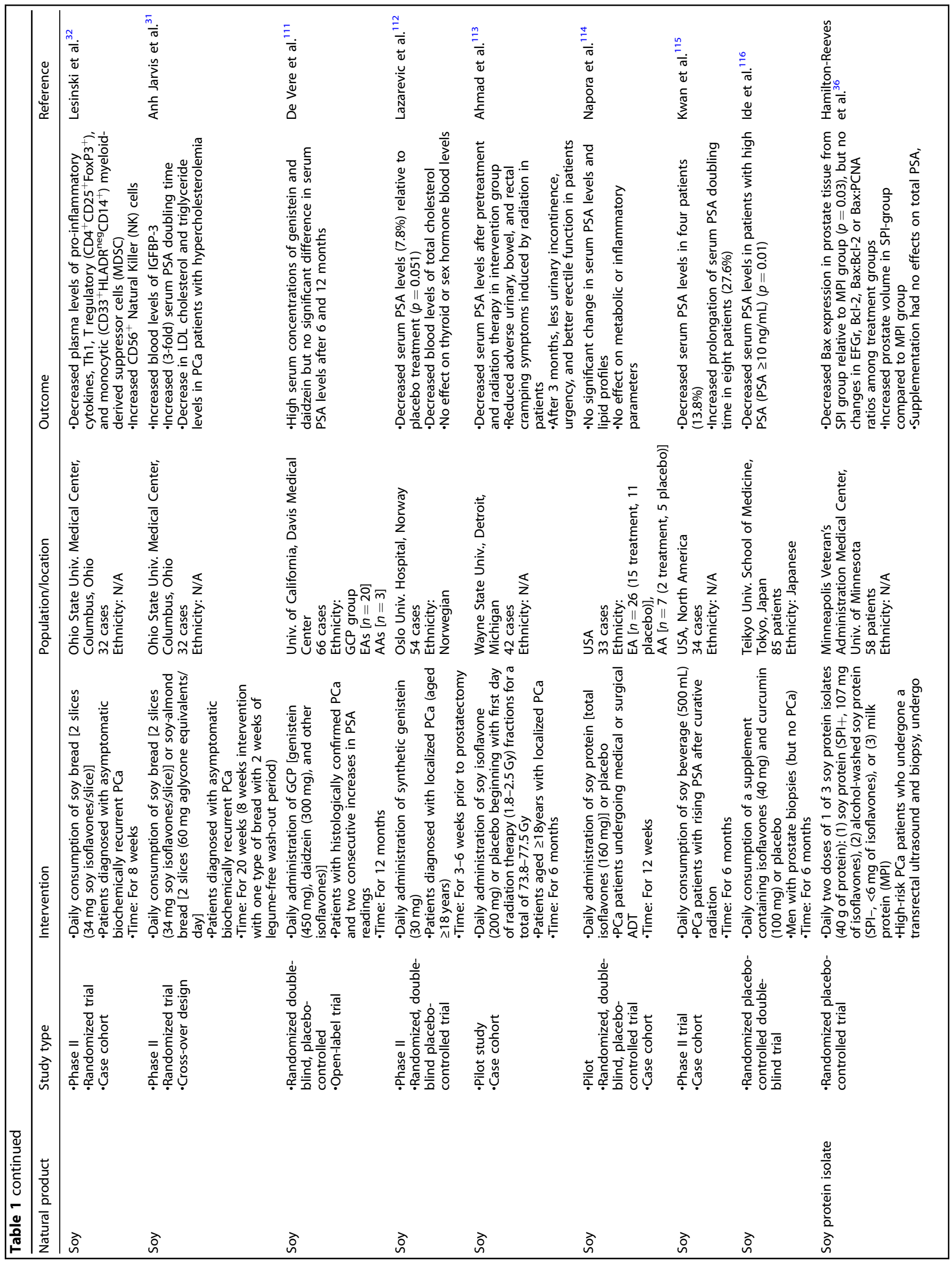




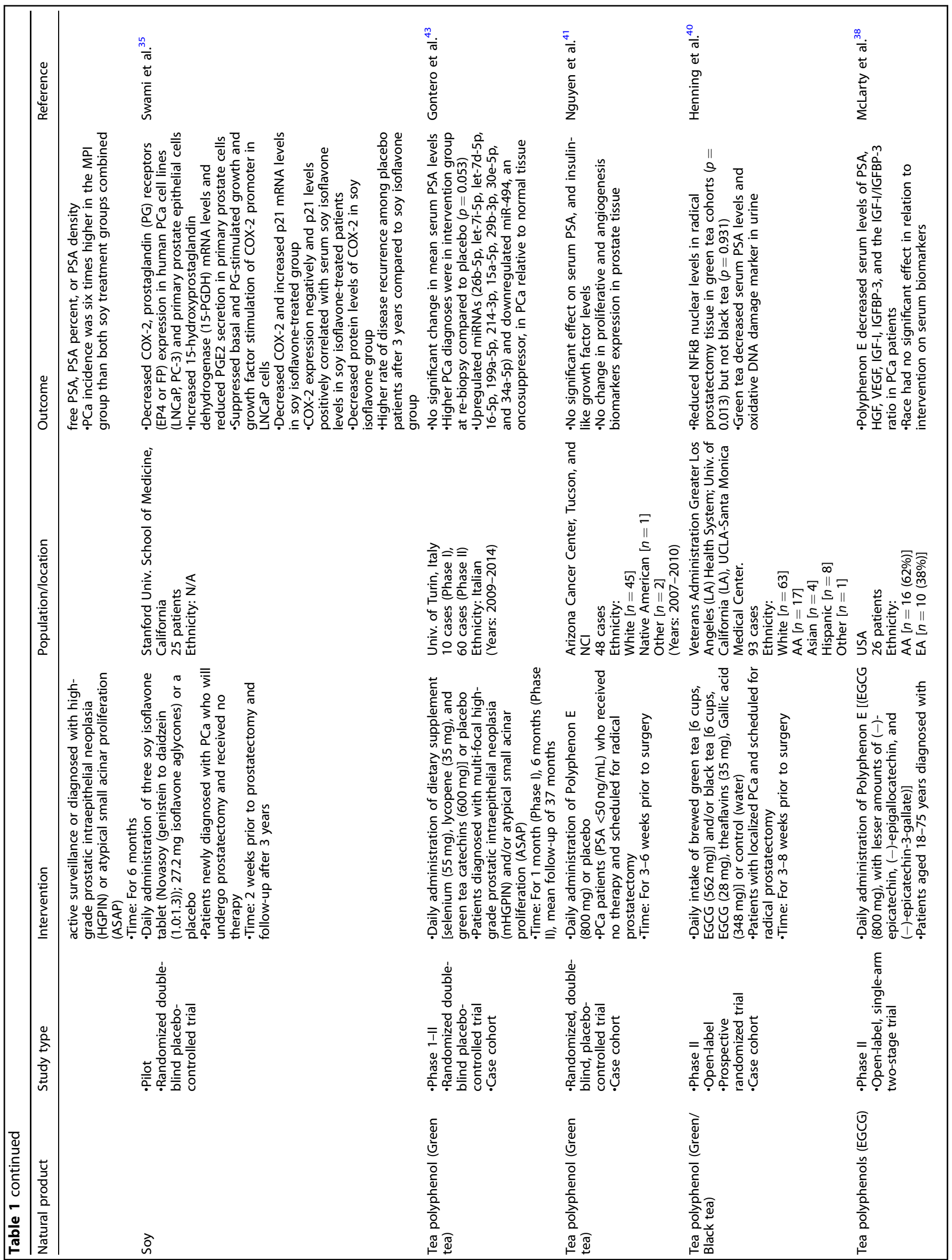


retinol also known as vitamin A. ${ }^{9}$ Epidemiologic and clinical evidence have shown an inverse relationship between dietary lycopene (including other carotenoids), PCa development and disease progression risk. ${ }^{9,10}$ In a randomized-controlled study, a significant decrease in median PSA levels among PCa patients in the lycopene intervention group relative to control subjects was observed. ${ }^{11}$ Based on non-metastatic PCa patients in the Cancer Prevention Study II Nutrition Cohort, prediagnosis and postdiagnosis dietary lycopene intake did not modify PCa-specific mortality ${ }^{12}$ however, lycopene intake higher than the median value was significantly associated with $59 \%$ lower hazard ratio among high-risk PCa patients. Notably, in a recent prospective study in patients from the Health Professionals Follow-Up cohort, over a 23-year follow-up, average tomato sauce intake was associated with a $46 \%$ reduction in risk of TMPRSS2-ERG-fusion positive PCa disease. ${ }^{13}$ High plasma levels of cis-lutein/zeaxanthin, all-trans-lycopene, and $\beta$-cryptoxanthin after 3 months corresponded to lower PSA levels at 3 and/or 6 months, respectively. ${ }^{14}$ High circulating levels of a-carotene, ${ }^{10,15} \beta$-carotene, and total carotenoids lowered PCa susceptibility; ${ }^{10}$ however, circulating levels of lycopene were associated with a non-significant reduction in $\mathrm{PCa}$ risk.

Although carotenoids have exhibited preventive effects against $\mathrm{PCa}$, other studies have reported little to no effect and/or antagonistic effect of carotenoid-associated nutraceuticals in PCa susceptibility. ${ }^{9,12,15-26}$ In a large epidemiologic study, lycopene intake was not associated to overall PCa risk; however, retinol, a biosynthesis product of several carotenoids, was linked to a $13 \%$ increase $(p=0.015)$ in risk. ${ }^{9}$ Similarly, in the SU.VI.MAX study, a double-blind placebo-controlled and randomized trial, $\beta$-carotene had no effect on hazard ratios associated with PCa among 5141 men. ${ }^{18}$ In a cross-sectional study, high serum lycopene levels and total PSA $>2.5 \mathrm{ng} / \mathrm{mL}$ were associated with a 1.49 -fold increase in PCa risk. ${ }^{15}$ Whereas, serum or plasma levels of retinol, $\beta$-carotene, $\beta$-cryptoxanthin, lutein and/or zeaxanthin, and total carotenoids did not significantly modify risk. ${ }^{15,19}$ Higher risk of PCa mortality was linked to $\beta$-carotene intervention in men relative to non-users in the Alpha-Tocopherol, Beta-Carotene Cancer Prevention (ATBC) Study $(n=29,133) .{ }^{26}$ In a prospective study, serum levels of acarotene, $\beta$-carotene, lycopene, retinol did not show a relationship with time to disease progression, treatment, PSA kinetics (i.e., PSA, PSA velocity) and adverse histology in patients with localized disease. $^{20}$ Likewise, another prospective study showed no association between lycopene and $\beta$-carotene serum levels and PCa risk in Australian men. ${ }^{21}$ In the PCa Prevention Trial (PCPT), dietary intake of carotenoid excluding lycopene had no effect on the risk of total incident symptomatic benign prostatic hyperplasia (BPH) among placebo arm participants after a 7-year follow-up ( $n$ $=4770) .{ }^{17}$ In the intervention arm of this study, lycopene also showed no significant association with BPH risk. Notably, carotenoid intervention and circulating levels in relation to $\mathrm{PCa}$ development and advanced disease have resulted in null findings in other reports. ${ }^{12,16,22-25,27}$

Lycopene supplementation has shown a modest impact on cellular death, cell cycle, growth, and oxidative stress signaling mechanisms. ${ }^{28,29}$ Recent studies included in this review showed no molecular changes related to other carotenoids. In a Phase II clinical trial, 6 months of lycopene intervention marginally reduced nuclear levels of proliferative marker MCM-2 and cell cycle regulator p27 in benign prostate tissue from patients diagnosed with high-grade prostatic intraepithelial neoplasia (PIN). ${ }^{28}$ After a 3-month lycopene or tomato product intervention, analysis of normal prostate tissue from low-risk PCa patients (Gleason sum $\leq 6$ ) showed apoptotic signaling and nuclear factor (erythroid derived-2) 2-mediated oxidative stress response as the top two ranked pathways altered by treatment compared to placebo. $^{29}$ 


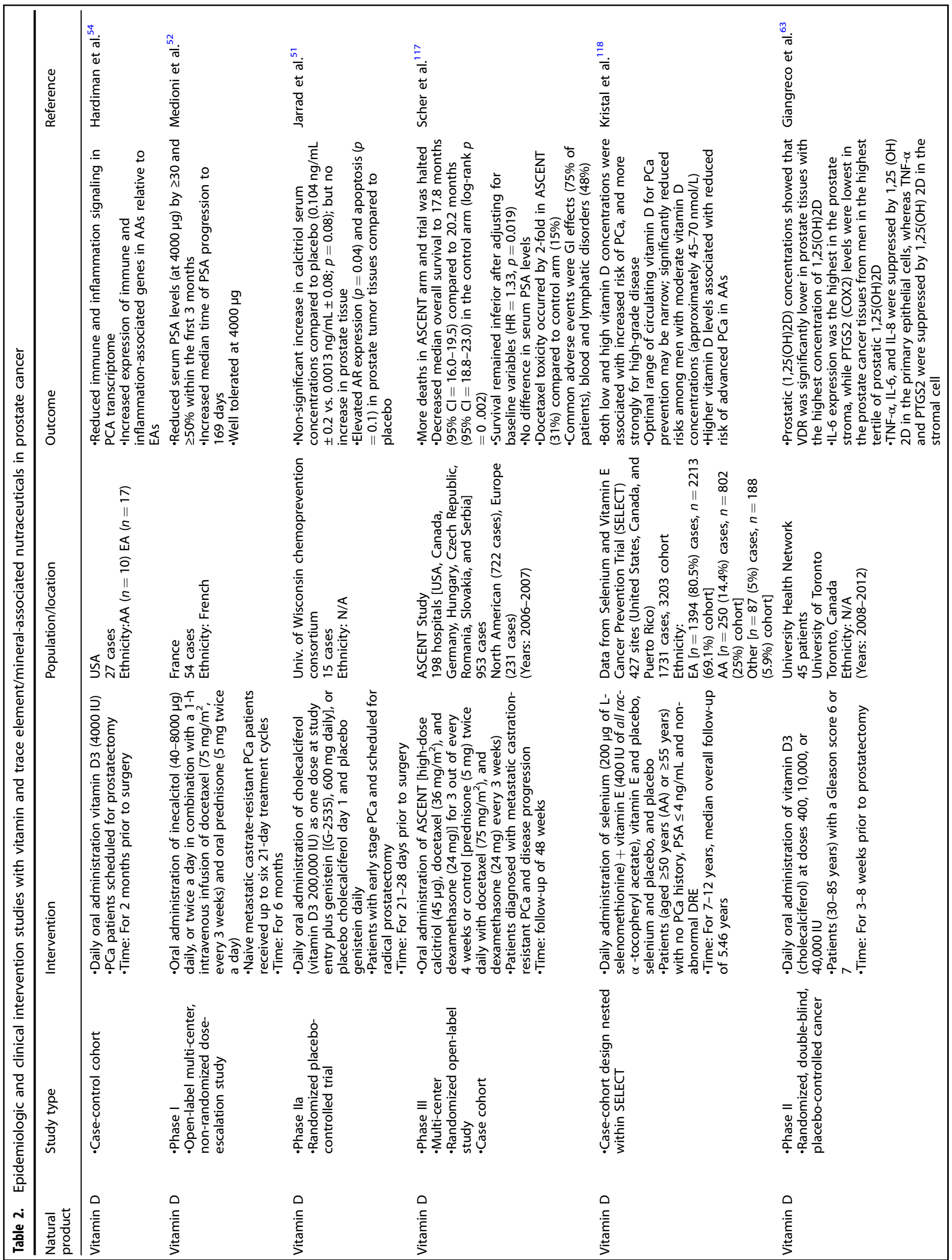




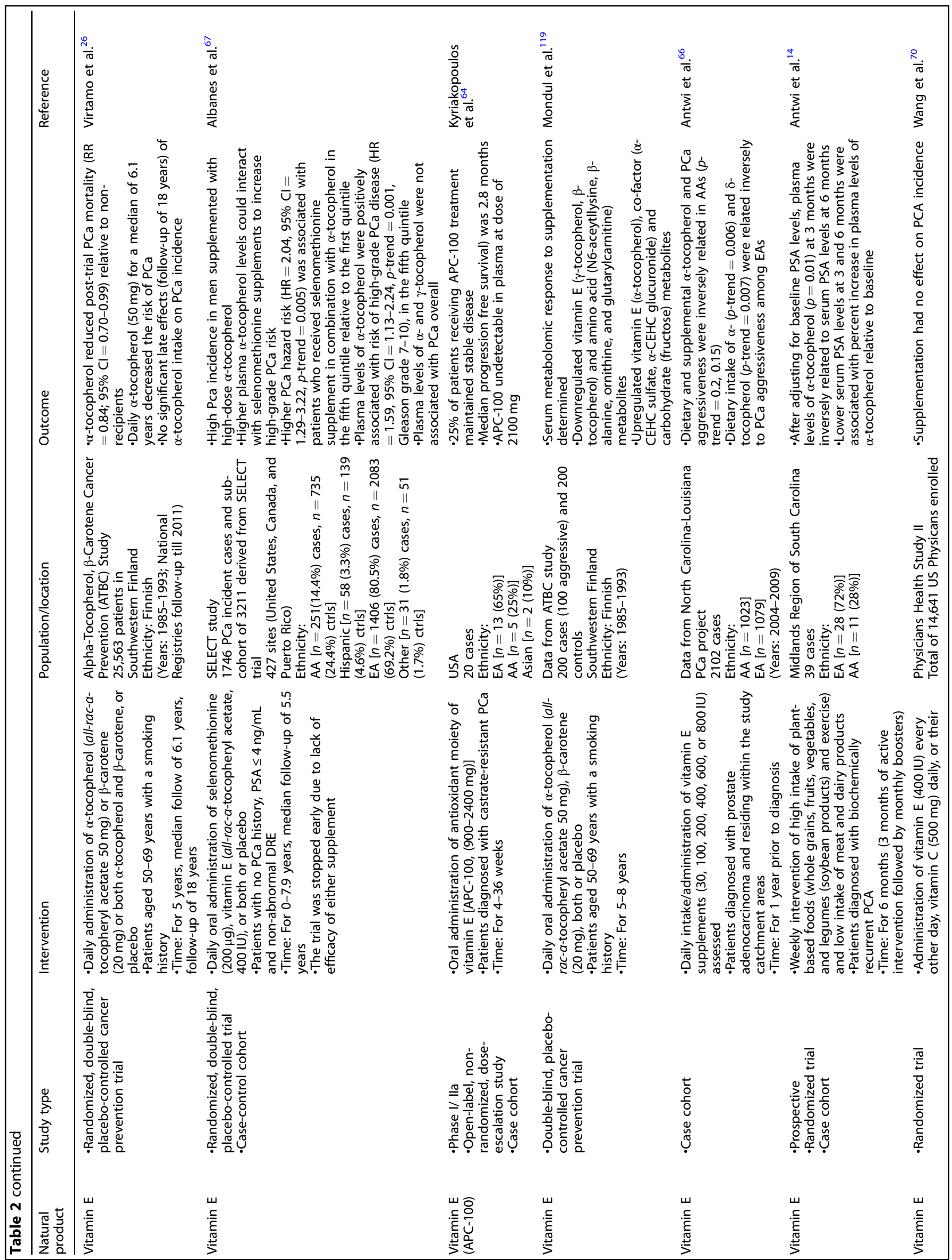




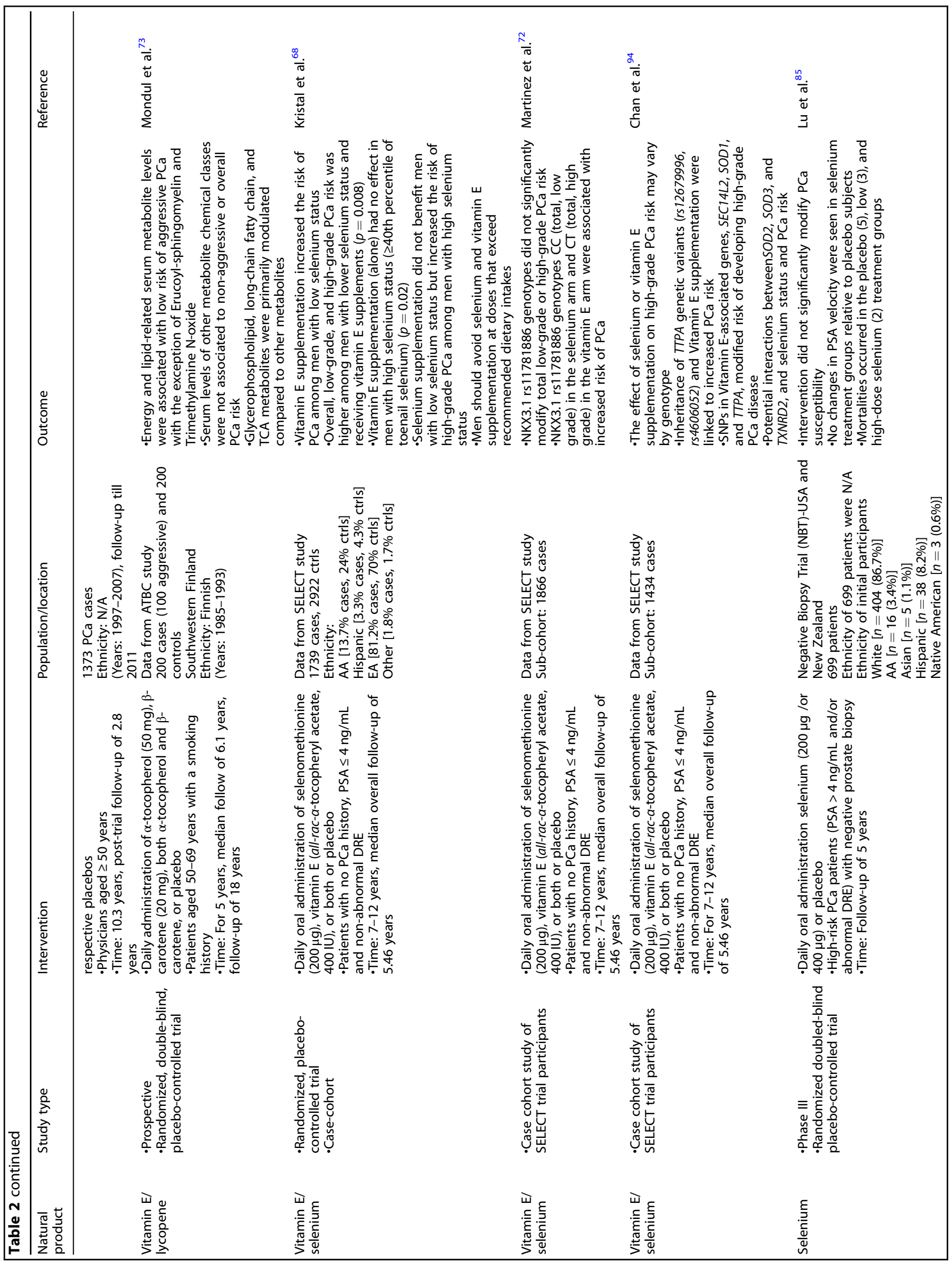




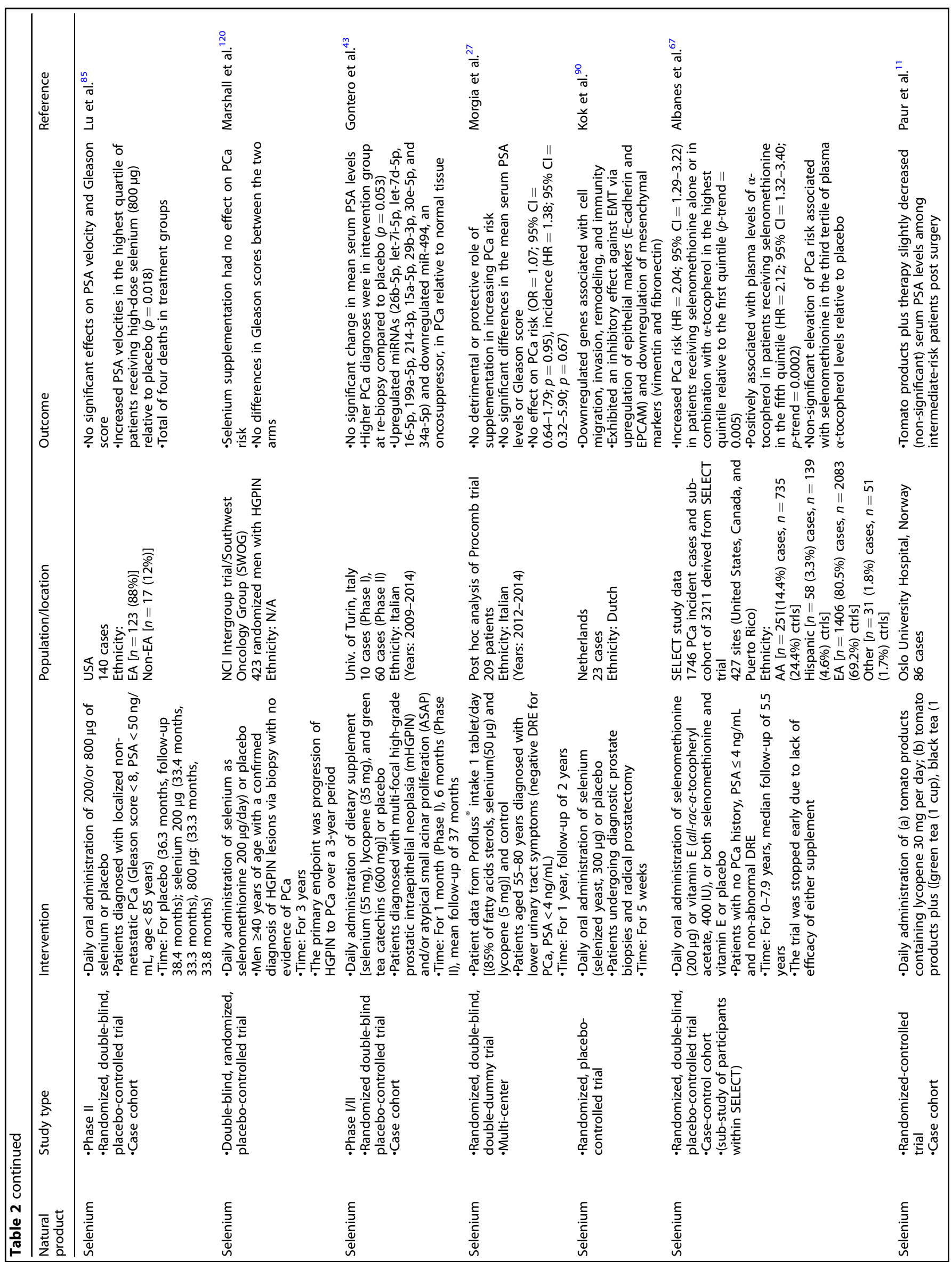




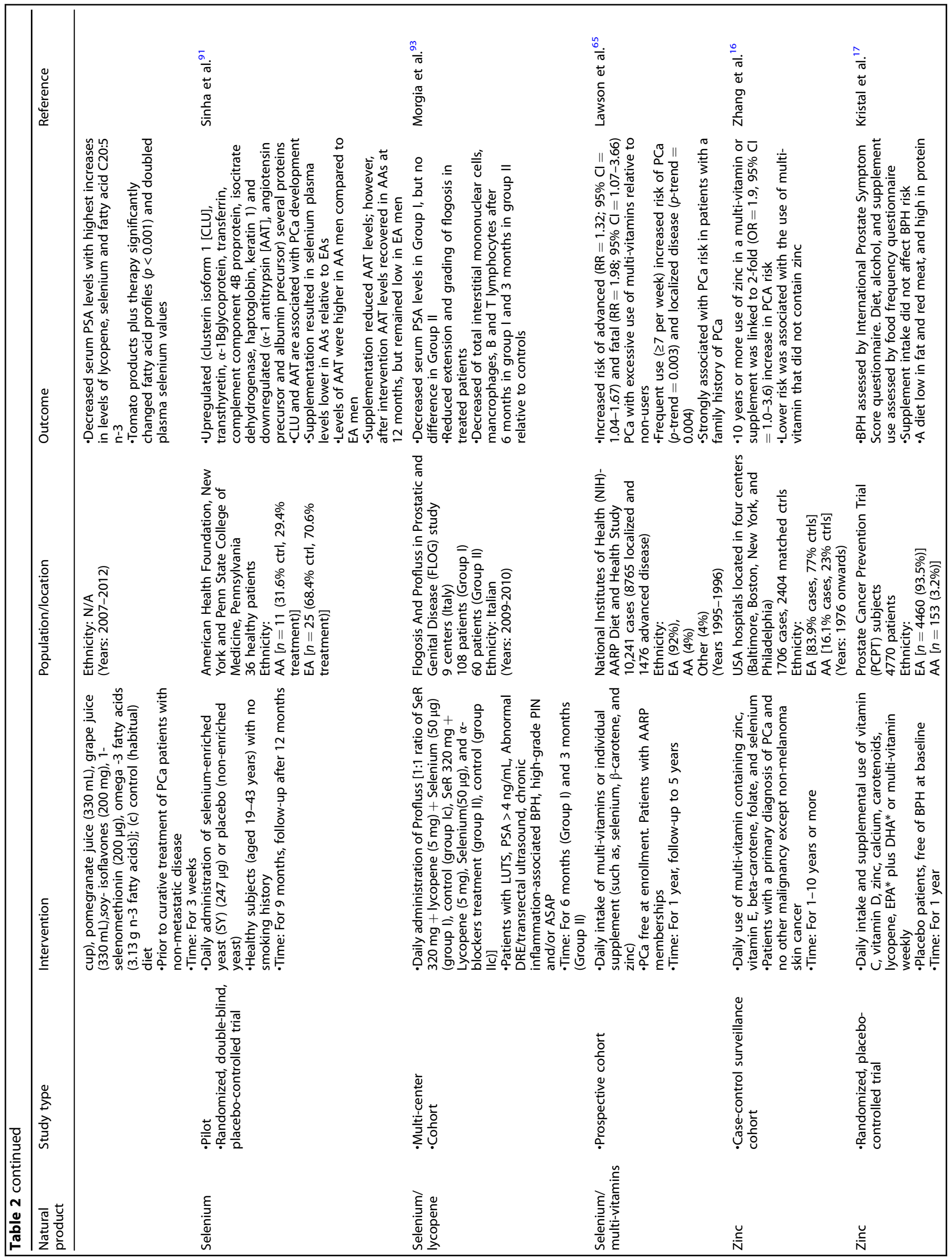


Soy isoflavones

Soy Isoflavones are members of the polyphenolic flavonoid family. ${ }^{30}$ These compounds are found in red clover, kudzu root, and soybeans, which are commonly used in Asian and African cuisines. Genistein, daizein, aglycones, equol and glycitein are the predominant isoflavones in soybean and soy-derived food products. Clinical studies on the efficacy of soy intervention have shown some benefits against PCa via its influence on insulin and inflammatory signaling. In a 20 -week phase II trial in asymptomatic PCa patients, consumption of soy-almond bread and standard soy bread contributed to a significant increase in IGFBP-3 and decrease in pro-inflammatory cytokines blood levels. ${ }^{31}$ Unfortunately, dietary soy intervention increased serum PSA levels and its doubling time after 126 days with a slight decrease in PSA velocity in PCa patients. Similar to the previous report, isoflavones have exhibited some immunomodulatory properties in the plasma of asymptomatic biochemically recurrent $\mathrm{PCa}$ patients. ${ }^{32}$ High plasma genistein concentrations $(>640.2 \mathrm{nmo} / \mathrm{L})$ were strongly linked to a $69 \%$ decrease in risk of developing PCa among Chinese patients. ${ }^{33}$ At the time of diagnosis, median levels of plasma genistein were significantly lower in $\mathrm{PCa}$ patients relative to controls. Short-term administration of isoflavone $(80 \mathrm{mg})$ for 6 weeks in patients also showed an inhibitory effect on cell cycle and apoptotic-associated signaling in prostate tumor tissue. ${ }^{34}$ However, isoflavone intervention did not affect serum levels of total testosterone, free testosterone, PSA, and total cholesterol in PCa patients.

Though limited reports have evaluated genomic evidence for the anti-cancer properties of soy isoflavones in PCa clinical trials, soy supplementation has exhibited some effects on inflammatory, apoptotic, and growth signals in PCa. ${ }^{31,35,36}$ In a pilot randomized double-blinded clinical study, soy isoflavones intervention altered the expression of COX-2, a major molecule in prostaglandin synthesis and cyclin kinase inhibitor p21 in PCa patient prostatectomy specimens. ${ }^{35}$ In the same study, genistein treatment also downregulated COX-2 in both LNCaP and PC-3 cell lines and upregulated 15-PGDH in primary PCa cells. In a 6-month clinical trial, soy protein intervention had no effect on proliferative, and apoptotic molecular markers (i.e., EGFR, Bax:Bcl-2, Bax:PCNA ratios) in high-risk and/or with low-grade PCa disease patients, but alcohol-washed soy protein intake reduced tissue levels of PCNA and Bax in patients relative to milk protein treatment. ${ }^{36}$

Green and black tea extracts

Green and black tea are extracted from the plant Camellia sinensis. (-)-Epigallocatechin-3-gallate (EGCG) is the most abundant and well-studied bioactive polyphenolic constituent of green tea with regard to its anti-cancer properties in several malignancies including $\mathrm{PCa}^{37}$ Theaflavin is the major bioactive polyphenol from black tea; however, it has not been well studied compared to EGCG. ${ }^{37}$ Many population studies have examined the efficacy of green tea in PCa chemoprevention. ${ }^{23,38-41}$ In a recent metaanalysis of 13 clinical studies, green tea catechins demonstrated protective effect against PCa risk. ${ }^{23}$ Concurrent consumption of green tea catechins and natural food products in an adjusted indirect comparison relative to six other natural compounds significantly reduced PCa susceptibility in men diagnosed with high-grade PIN. Daily administration of Polyphenon E (an enriched green tea polyphenol extract) containing $800 \mathrm{mg}$ of EGCG prior to a radical prostatectomy significantly reduced serum levels of PSA in men with cancer-positive prostate biopsies. ${ }^{38}$ However, in another study, Polyphenon E intake showed no significant effects on the serum levels of PSA, insulin-like growth factor, proliferation, and angiogenesis in the prostate tissue of $\mathrm{PCa}$ patients after 3-6 weeks. $^{41}$ The efficacy of green tea was also evaluated in patients with localized PCa $(n=199)$ and receiving active surveillance or watchful waiting as clinical management 
treatment. Oral administration of a capsule containing nutraceuticals, pomegranate, green tea, broccoli, and turmeric for 6 months significantly decreased median PSA percentage levels in patients, regardless of clinical management relative to the placebo group. ${ }^{39}$ Unfortunately, this intervention did not alter Gleason grades in patients.

Tea polyphenols have modulated several molecular signatures in PCa patients. Green tea intake reduced nuclear NFkB in radical prostatectomy tissue and PSA levels in PCa patients compared to black tea and control treatments 3-8 weeks prior to surgical therapy. ${ }^{40}$ Growth factor signaling mediators, HGF, VEGF, IGF, and IGF/IGFBP-3, ratio was decreased in men with PCa. ${ }^{38,41-43}$ Although expression of cell proliferative, apoptotic, and angiogenic markers was not changed by green tea intervention after 3-6 weeks prior to surgery, serum levels of PSA, IGF, and DNA oxidative stress in leukocytes were decreased by treatment in patients. ${ }^{41}$ Also, green and black tea reduced the proliferation of LNCaP PCa cells. ${ }^{42}$ Green tea in a combinatorial nutraceutical intervention promoted oncogenic-related miRNAs in PCa including miR-92-3p that targets PTEN and androgen-regulated miR$125-5$ p. ${ }^{43}$

\section{Vitamin D}

Vitamin D is a fat-soluble nutraceutical found in dairy, flour, and fortified food products. ${ }^{44}$ It has five isoforms, which include ergocalciferol with lumisterol (D1), ergocalciferol (D2), cholecalciferol (D3), 22-dihydroergocalciferol (D4), and sitocalciferol (D5). Its biosynthesis occurs in the skin in response to solar ultraviolet $B$ radiation exposure. In the body, Vitamin $D$ primarily circulates as 25 -hydroxyvitamin $\mathrm{D}[25(\mathrm{OH}) \mathrm{D}]$ and is converted by $1 \mathrm{a}$-hydroxylase into its active form, 1,25-dihydroxyvitamin D [1,25(OH)2D]. Calcium and bone homeostasis are closely dependent on Vitamin D bioavailability. Vitamin D deficiency is linked to elevated susceptibility of PCa development and aggressive disease. Both clinical and other population-based studies have comprehensively evaluated the role of dietary vitamin $D$ as a preventive therapy to reduce disease development and complimentary agent to accepted clinical treatments for PCa. Overall, dietary vitamin D levels have an inverse association to PCa development and disease progression. ${ }^{45,46}$ Vitamin D deficiency $(<20 \mathrm{ng} / \mathrm{mL})$ alone increases the risk of PCa development and aggressive PCa in certain subpopulations. ${ }^{47}$ In two large nested case-control studies, vitamin $D$ intervention decreased the risk of developing $\mathrm{PCa}$ among patients with high Gleason scores. ${ }^{48}$ In a cross-sectional study, dietary Vitamin D intake showed an inverse association with aggressive PCa (699 cases and 958 controls). ${ }^{46}$ Additionally, high serum levels of vitamin D were correlated with a decrease in PCa risk among men. ${ }^{48}$

Strong expression of calcium-sensing receptor was also related to lethal progression in PCa tumors with low vitamin D receptor (VDR) levels, whereas high VDR-expressing tumors were not linked to disease progression. ${ }^{49}$ In the Malmo Diet and Cancer Study, low PCa mortality was linked to $25(\mathrm{OH}) \mathrm{D}$ concentrations (>85 nmol/ L), ${ }^{50}$ but lower levels of this metabolite $(<50 \mathrm{nmol} / \mathrm{L})$ increased PCa-specific mortality risk in another study. ${ }^{45}$ In a Phase II clinical trial, daily combination therapy of cholecalciferol/vitamin D3 $(200,000 \mathrm{IU})$ and G-2535 (genistein, $600 \mathrm{mg}$ ) increased serum levels of calcitriol but this was not reflected in prostate tissues. ${ }^{51}$ Although no increase in calcitrol was observed in prostate, this treatment enhanced androgen receptor (AR) expression and proapoptotic effects in prostate tumor tissue compared to placebocontrol subjects. In naive metastatic castrate-resistant PCa, a VDR agonist, Inecalcitol, used in combination with docetaxel and prednisone effectively reduced PSA levels in $76 \%$ of patients. ${ }^{52}$ Furthermore, high expression of inflammatory markers, serum Creactive protein (CRP), and interleukin IL-8 were inversely related to $25-(\mathrm{OH}) \mathrm{D}$ levels in PCa patients. ${ }^{53}$ Vitamin D3 supplementation
(4000 IU daily) promoted higher levels of immune- and inflammation-related genes in US men within 2 months prior to a prostatectomy. ${ }^{54}$ In a nested case-control study of Sweden PCa patients (943 cases), high risk was linked to men in the highest quartile of vitamin $D(\geq 103 \mathrm{nmol} / \mathrm{L})$ with a moderate Gleason score (7) or high serum parathyroid hormone (PTH) levels $(\geq 3.74 \mathrm{pmol} / \mathrm{L})$ as well as men in the highest quartiles of both $\mathrm{PTH}$ and calcium $(\geq 2.38 \mathrm{mmol} / \mathrm{L}) .{ }^{55}$ Lower risk of aggressive and non-aggressive disease was significantly linked to men in the 4th quartile for PTH with low serum levels of Vitamin D $(<50 \mathrm{nmol} / \mathrm{L})$. Unfortunately, several population studies have depicted null relationships between vitamin $D$ concentration levels and/or supplementation and PCa susceptibility in median-sized cohorts. ${ }^{17,22,48,56-61}$

Vitamin D treatment has shown some anti-inflammatory and hormone-related molecular changes in PCa. ${ }^{51,53,62,63}$ In Taiwanese PCa patients, genetic variants, HFE rs9393682, and TUSC-3 rs1378033 were associated with time to progression in localized disease and low risk of advanced PCa for patients undergoing androgen deprivation therapy. ${ }^{62}$ Furthermore, in vitro studies revealed 1,25-Vitamin D downregulated HFE and when silenced HFE impedes cell proliferation and wound healing. Low expression of TUSC-3 was shown to correspond with poor PCa prognosis in patients, and TUSC-3 silencing enhanced cell migration and growth. ${ }^{62}$ However, 1,25-Vitamin D strongly induced the expression of TUSC-3 in PCa cells. In a Phase II clinical trial, PCa patients with the highest serum levels of prostatic 1,25(OH)2D also had low COX-2 levels, but exhibited high IL-6 levels in their stroma tissue. ${ }^{63}$ Interestingly, 1,25 (OH)2D treatment suppressed TNF-a, IL-6, and IL-8 levels in primary epithelial cells, but only TNF-a and COX-2 levels were downregulated in stromal cells. Similarly, circulating levels of $25(\mathrm{OH}) \mathrm{D}$ negatively correlated with pro-inflammatory markers, serum CRP, and IL-8, but NFKB p65-positive cells were elevated in PCa patients. ${ }^{53}$ In early stage PCa, high-dose cholecalciferol and genistein (G-2535) intervention induced AR expression in patient tumor tissue but not benign tissue relative to placebo controls. ${ }^{51}$

\section{Vitamin $\mathrm{E}$}

The protective effect of nutraceutical, Vitamin E, in PCa has been widely studied in published reports and clinical trials. ${ }^{9}$ Tocopherols $(a, \gamma$, and $\delta)$ possess vitamin E activity and are the most studied vitamin E bioactive constituents in PCa. Vitamin E is fat soluble and found in a variety of foods, such as nuts, seeds, and vegetable oil. Both European and Western diets have a high content of tocopherols. European diets mainly include a-tocopherol, whereas $\gamma$-tocopherol is generally present in the Western diets. In a pooled study including 15 cohorts (11,239 cases, 18,541 controls), atocopherol consumption was associated with a decrease in risk of PCa overall and aggressive disease susceptibility. ${ }^{9}$ In a small clinical trial, castrate-resistant PCa patients taking APC-100, an antioxidant moiety of a-tocopherol, maintained stable disease and median progression-free survival of 2.8 months. $^{64}$ In a 6-month clinical trial, high a-tocopherol levels were inversely related to serum PSA levels in biochemical recurrent PCa patients $(n=39){ }^{14}$ In contrast, other studies have shown negative effects associated with vitamin $\mathrm{E}$ intervention or its circulating plasma levels in $\mathrm{PCa}$ patients. PCa risk was slightly increased in the National Institutes of Health (NIH)-AARP Diet and Health Study due to a high frequency ( $>7$ times per week) of dietary Vitamin E. ${ }^{65}$ High PCa risk estimates were associated with patients who used a dosage of $800 \mathrm{IU}$ per day regardless of frequency. Furthermore, patients with family history of PCa and taking frequent multi-vitamin supplementation (>7 times per week) had a 2.48 and 16.41-fold increase in susceptibility of advanced and fatal PCa disease, respectively. In another report, dietary intake of both a-tocopherols and $\delta$ tocopherols reduced risk of PCa in European-American patients. ${ }^{66}$ 
Furthermore, positive plasma a-tocopherol levels were also linked to high-grade PCa disease (Gleason grade 7-10). ${ }^{67}$ However, vitamin $E$ intervention combined with low selenium status increased $\mathrm{PCa}$ susceptibility in men. ${ }^{68}$ Although vitamin $E$ supplementation has some negative effects in $\mathrm{PCa}$, some large population studies have observed null findings in relation to $\mathrm{BPH}$ and PCa. ${ }^{17,22,66,68-70}$

Vitamin E intervention, supplementation, and associated genetic variants have been shown to modulate PCa susceptibility among men. ${ }^{71-74}$ In the multi-center Prostate, Lung, Colorectal, and Ovarian Cancer Screening Trial, rs964184 variant GG genotype located near genes, BUD13, ZNF259, and APOA5, which plays a role in Vitamin $\mathrm{E}$ metabolism, showed a protective role against PCa risk with the inheritance of two or more minor alleles. ${ }^{71}$ However, other clinical trials have identified variants such as NKX3.1 rs11781886 linked high risk of developing advanced PCa disease in the presence of Vitamin E intervention. ${ }^{72}$ In the ATBC study cohort, serum levels of tricarboxylic acid cycle, long-chain fatty acid, and glycerophospholipid metabolites were strongly associated with low risk of aggressive PCa with the exception of metabolites, thyroxine, and trimethylamine oxide. ${ }^{73}$ However, these metabolites had no significant interaction with a-tocopherol supplementation. Vitamin E-related transcripts involved in the transport of vitamin E influence PCa susceptibility as well. In a clinical study by Bauer and colleagues (2013), circulating levels of a-tocopherol or $\gamma$-tocopherol were associated with disease recurrence; however, superoxide dismutase enzyme 3 (SOD3) rs699473 variant were linked to high-grade PCa, but SOD1 (rs17884057, rs9967983) and SOD2 (rs4880) variants were protective against disease recurrence among men $(n=573) .^{74}$ Additionally, SOD1 rs17884057 variant and circulating a-tocopherol levels had a significant interaction with high-grade $\mathrm{PCa}$, but did not remain significant in the highest quartile of a-tocopherol.

\section{Zinc}

Normal prostatic tissue exhibits the highest levels of the mineral zinc compared to any soft tissue in the body. ${ }^{75}$ In earlier epidemiologic studies, dietary zinc demonstrated a protective effect in individuals diagnosed with advanced PCa susceptibility. However, recent reports oppose the preventive role of dietary zinc and contribute to mix findings on the relationship of zinc consumption and PCa risk. Long-term dietary zinc from multivitamins or other supplements in PCa patients showed a nonsignificant 2.0-fold elevation in PCa risk in the US hospital-based Case-Control Surveillance Study (1706 cases, 2404 matched controls). ${ }^{16}$ Epidemiologic evidence has also revealed an interaction between dietary zinc intake and cadmium exposure in relation to PSA levels. In the 2001-2002 National Health and Nutrition Examination Survey, dietary zinc intake less than the median level $(<12.67 \mathrm{mg} /$ day) was linked to an increase in creatinine cadmium exposure $(1 \mu \mathrm{g} / \mathrm{g}) .^{76}$ Cadmium is a nonessential heavy metal that is one of the naturally occurring composites in zinc. This relationship induced a $35 \%$ increase in PSA levels in patients. However, this association between cadmium exposure and PSA levels disappears at higher levels of dietary zinc intake. ${ }^{76}$ During the 7 -year follow-up period in the PCPT study, ${ }^{17}$ dietary and total zinc intake decreased the susceptibility to develop total symptomatic BPH incidence among the placebo population in the second, fourth, and fifth quintiles. ${ }^{17}$ In the VITAL study, Gonzalez and associates showed that dietary zinc intake over a 10-year period had a non-significant decrease in overall PCa and advanced disease (regional/distant) mortality risk. $^{77}$

Other studies have shown either null and/or possible harmful effects of dietary zinc intake and PCa and/or other associated conditions. ${ }^{16,17,22,65,77-79}$ Varying levels of dietary zinc have been linked to potential PCa risk. Two studies showed that excessive use of multi-vitamin with zinc supplements was associated with high risk of PCa. ${ }^{16,65}$ In the NIH-AARP Diet and Health Study, excessive use of multi-vitamin including zinc was associated with a 4.36-fold increase in the risk of developing fatal PCa among 10,241 men (8765 localized and 1476 advanced cases). ${ }^{65}$ In other studies including the Case-Control Surveillance Study and SU.VI. MAX study, non-significant associations were shown between zinc intake and/or supplementation and circulating levels with PCa susceptibility among European-American, African-American, and Mexican-American men. ${ }^{16,22,79,80}$

Unfortunately, current clinical studies have not evaluated the molecular effects of zinc intervention in PCa. However, two studies have evaluated some aspects of zinc homeostasis affected in PCa. It is well known that zinc levels as well as the expression of zinc transporters are low in PCa and this may impair zinc absorption in the body. ${ }^{81}$ Zinc transporters, hZIP1 and hZIP2, were underexpressed in malignant prostate tissue compared to surrounding normal tissue. ${ }^{81}$ In another study, miR-182 expression in prostate tissue was higher in all PCa cases, but miRs-182 and miRs-346 expression were inversely related to hZIP1 levels in EuropeanAmerican men only. ${ }^{82}$ Overexpression of miRs-183, miRs-96, and miRs-182 reduced intracellular zinc levels and uptake in primary prostatic epithelial cells. Despite recent reports that suggest a possible antagonistic role for zinc in PCa, additional studies are still needed to fully evaluate the efficacy of dietary zinc or supplementation in patients due to limited literature on the molecular and clinical impact of this agent on PCa.

\section{Selenium}

Changes in the physiological levels of the mineral selenium may influence biochemical and metabolic processes in PCa. ${ }^{83}$ Significantly low levels of selenium have been detected in malignant prostatic tissue compared to BPH specimens. ${ }^{84}$ Dietary selenium intervention has been linked to null findings for PCa susceptibility. ${ }^{16,17,22-25,27,85}$ In two meta-analysis studies, selenium intake was related to an increase (though not significant) in PCa risk. ${ }^{23,86}$ In a pooled study of prospective cohorts, the daily intake of 37 supplements including selenium (49-90 $\mu \mathrm{g})$ did not modify PCa risk in British men. ${ }^{22}$ In the Italian cohort for the Procomb trial (ISRCTN78639965), selenium intervention for 2 years did not significantly change PSA levels, PCa susceptibility, or exhibit a strong association with high PCa mortality risk. ${ }^{27}$ In a nested case control study based on the Physicians' Health Study and Health Professionals Follow-Up Study cohorts, circulating selenium levels did not significantly modify PCa susceptibility. ${ }^{24}$ No association was observed between circulating levels of selenium and PCa susceptibility considering TMPRSS2-ERG-fusion positive or negative cancer stratification relative to control subjects (370 cases, 2740 controls). Over the course of 3-5 years, selenium supplementation of 200 or $400 \mu \mathrm{g}$ did not affect PSA velocity or risk of PCa mortality in high-risk patients $(n=699)$ in a Phase III randomized, doubleblinded placebo-controlled, multi-center trial. ${ }^{85}$

Interestingly, meta-analysis of 17 western population-based studies showed serum selenium was inversely related to PCa susceptibility in individual study cohorts. ${ }^{86}$ Arsenic exposure and plasma selenium levels influence PCa susceptibility in patients. In a case-control study on Taiwanese patients (318 cases and 318 controls), low plasma selenium $(\leq 28.06 \mu \mathrm{g} / \mathrm{dL})$ and high urinary arsenic concentration ( $>29.28 \mu \mathrm{g} / \mathrm{L}$ ) were associated with elevated PCa risk in multi-variate analyses and significantly interacted with PSA levels $(\geq 20 \mathrm{ng} / \mathrm{mL}){ }^{87}$ In a meta-analysis of 15 prospective studies on PCa patients (4527 cases and 6021 controls), high selenium plasma levels were associated with a lower risk of aggressive $\mathrm{PCa}$ and selenium nail content was inversely related to PCa risk, but not for blood selenium. ${ }^{88}$ High toenail selenium levels and selenoprotein $\mathrm{P}$, a major selenium transporter, variants were associated with reduced advanced PCa risk. ${ }^{89}$ In a randomized- 
controlled trial, tomato product intervention consisting of selenium slightly increased PSA levels among patients with intermediate risk of PCa $(n=86){ }^{11}$ During this intervention, patients with high levels of lycopene, selenium, and fatty acid C20:5 n-3 combined were linked to a large decrease in serum PSA levels. Selenium intervention has also triggered some inconsistent molecular alterations in PCa. Selenium intervention $(300 \mu \mathrm{g})$ triggered a reversal of epithelial-to-mesenchymal transition (EMT) via upregulation of epithelial markers and lower expression of mesenchymal-related genes in a Dutch population compared to those receiving the yeast placebo. $^{90}$ Selenium-enriched yeast reduced levels of proteins related to anti-apoptosis (clusterin isoform 1), iron transport/homeostasis (transthyretin, haptoglobin), oncogenesis (a-1B-glycoprotein), inflammatory response (complement component $4 \mathrm{~B}$ proprotein), oxidative stress (keratin 1), NADH metabolic process (isocitrate dehydrogenase) in healthy African-American and European-American male subjects in a randomized, double-blinded, placebo-controlled clinical trial. ${ }^{91}$ However, selenium upregulated levels of $\mathrm{a}-1$ antitrypsin, a protein involved in hypoxia response, angiotensin precursor, negative regulator of cell growth and proliferation, and albumin, a negative regulator of apoptosis. Seven out of the 11 proteins altered by selenium-enriched yeast play a role in cancer; a-1B-glycoprotein, transferrin, haptoglobin, transthyretin, a-1 antitrypsin, and angiotensin precursor. ${ }^{91}$ In a nested case-control study evaluating the effect of selenium and dietary glucosinolate intake, glutathione peroxidase activity exhibited no significant association to benign hyperplasia risk among men $(n=325) .{ }^{92}$ Profluss, a mixture of selenium, lycopene, and saw palmetto tree berries, decreased tissue levels of total interstitial mononuclear cells, B lymphocytes, T lymphocytes, and macrophages after 6 and 3 months in patients with PIN/Atypical small acinar proliferation (ASAP) and BPH, respectively. ${ }^{93}$ Six months of selenium administration combined with lycopene and green tea extract in patients with high-grade PIN and/or ASAP resulted in the upregulation of oncogenic microRNAs and decreased levels of miR-494, which plays a suppressive role in PCa. ${ }^{43}$ Inheritance of selenoprotein-associated variants were related to high-grade, ${ }^{94}$ susceptibility, and disease recurrence in PCa patients, but lost significance after adjustment for multiple comparisons. ${ }^{95,96}$

Other studies have demonstrated a non-protective role of selenium in PCa development and progression. ${ }^{43,65,67,85,96}$ High selenium status in men receiving vitamin $E$ supplements was linked to an increase in low-grade and high-grade disease development risk for PCa. ${ }^{85}$ Also, multi-vitamin use ( $>7$ times/ week) including dietary selenium was linked to an increase in the susceptibility of PCa. ${ }^{65}$ Interestingly, a large elevation of 5.8 -fold was related to PCa risk and dietary selenium use among fatal cases, but the effect was modest. In a double-blinded randomized clinical study, chemopreventive treatment including high-dose selenium in men $(n=30)$ diagnosed with multi-focal HGPIN and/ or ASAP had a higher frequency of PCa incidences after 6 months compared to placebo patients $(n=30){ }^{43}$ In the SELECT trial, a strong relationship between administration of selenomethionine, a type of selenium supplement, and higher plasma levels of atocopherol in men potentially promoted higher PCa hazard risk ratios. $^{67}$ Selenomethionine alone and any selenomethionine supplementation was linked to higher PCa mortality risk among men with high Gleason scores $(\geq 7)$. Moreover, high mortality risk in patients with the highest plasma levels of a-tocopherol and less than 3 year follow-up to diagnosis was associated with selenomethionine alone, a-tocopherol and selenomethionine or any selenomethionine supplementation.

Nutraceutical efficacy in diverse populations

To date, majority of nutraceutical chemoprevention/intervention studies have been evaluated in populations of European ancestry.
Commonly, higher PCa incidence and fatalities rates have been linked to men with African ancestry. Unfortunately, only a small percentage of studies have examined the effect of nutraceutical intervention on PCa development and progression, and disease traits in diverse populations as shown in Tables 1 and 2. Thus, current clinical and epidemiological studies lack evidence on how effective nutraceutical agents are against PCa in diverse populations. Of the limited studies, majority of these reports have focused on lycopene, selenium Vitamin D, and E supplementation or their circulating plasma levels in relation to PCa risk among men of African, Asian, Latino, and Native Hawaiian descent. In a large prospective cohort study (2015), selenium was related to PCa risk, but adjustment for both selenium and lycopene intake was associated with an increase in risk among Native Hawaiian, European, African, Japanese, and Latino-American men on a $1000 \mathrm{kcal}$ daily diet. ${ }^{25}$ Selenium in African-American patients was linked to high risk of localized, advanced disease, and PCa fatality, whereas Latino men were only linked to localized and low-grade disease. Low PCa risk was associated with high legume intake $(\geq 28.2 \mathrm{~g})$ in Latino men as well. ${ }^{25}$ Selenium had no effect on overall PCa risk regardless of ethnicity. However in the Selenium and Vitamin E Cancer Prevention trial, total, low-grade, and highgrade $\mathrm{PCa}$ incidences were higher among African-American men. ${ }^{97}$ Furthermore, African-American men with body mass index of 35 had a 2-fold PCa mortality risk. ${ }^{91}$

The effect of carotenoid circulating patterns and intervention on PCa differs slightly in different ethnic groups. As previously mentioned, adjustment for lycopene intake combined with selenium intake in a multi-ethnic cohort was related to PCa risk, but not when taken alone. ${ }^{25}$ Plasma levels of cis-lutein/zeaxanthin, $\beta$-cryptoxanthin, and all-trans-lycopene inversely related to biochemical recurrence in a cohort including African-American men, but in a later study dietary lycopene and $\beta$-cryptoxanthin were inversely related to aggressive PCa disease among European and African-Americans, respectively. ${ }^{98}$ Moreover, higher levels of a-carotene and lycopene (cis + trans) were observed in EuropeanAmerican men compared to their African-American counterparts. African-Caribbean men had higher circulating serum levels of acarotenes and $\beta$-carotenes and lutein/zeaxanthin, but lower lycopene/retinol levels due to their dietary intake compared to African-Americans. ${ }^{99}$ Moreover, high PSA levels were marginally linked to low retinol serum levels in Caribbean men. Based on the aforementioned studies, $\beta$-cryptoxanthin may have a protective role against PCa in African-American men. The data is suggestive that high a-carotene serum levels may contribute to $\mathrm{PCa}$ development in European-American and Caribbean men; however, due to limited epidemiological and clinical data more studies are needed.

Vitamin D deficiency in African-American men has been linked to high risk of $\mathrm{PCa}$ and aggressive disease. ${ }^{47}$ Moreover, low plasma 25(OH)D levels in African-Americans was significantly related to PCa aggressiveness, while high calcium intake elevated risk. ${ }^{48}$ However, high dietary Vitamin D intake has been shown to reduce aggressive PCa risk in African-Americans, but this effect was not seen in European-Americans. ${ }^{46}$ Levels of vitamin D metabolites, 25 $(\mathrm{OH}) \mathrm{D}$ and $1,25(\mathrm{OH}) 2 \mathrm{D}$, vary greatly in PCa; serum levels of $25(\mathrm{OH})$ $\mathrm{D}$ are lower, but prostate tissue levels of 1,25(OH)2D are higher in African-Americans compared with European-Americans. ${ }^{100}$ Also, vitamin $\mathrm{D}$ binding protein expression negatively correlated to 25 $(\mathrm{OH}) \mathrm{D}$ serum levels in African-Americans. Although, AfricanAmerican PCa patients have low serum levels of $25(\mathrm{OH}) \mathrm{D}$, high circulating levels of $25(\mathrm{OH}) \mathrm{D}(34.27-93.20 \mathrm{ng} / \mathrm{mL})$ are linked to an increase in PCa risk among Jamaican men. ${ }^{48}$ Taken together, the previous studies implicate a possible antagonistic effect of $25(\mathrm{OH})$ $\mathrm{D}$ on PCa development in Caribbean men, but protective role in African-Americans, which warrant additional studies in these populations. 
Two major zinc transporters, hZIP1 and hZIP2, have been evaluated in malignant prostate tissue from European-American and African-American men. ${ }^{81}$ Very low levels of both transporters were observed in $92.8 \%$ of African-American PCa specimens. However, higher levels of zinc transporters were associated with high-grade prostate carcinoma in European-American men compared to African-Americans. In another study, miR-182 expression was higher in PCa tissue overall regardless of race, but miRs-182 and miRs-346 expression were inversely related to hZIP1 levels in European-American men only. ${ }^{82}$ Overexpression of miRs-183, miRs-96, and miRs-182 reduced intracellular zinc levels and uptake in primary prostatic epithelial cells. However, no differences were observed for hZIP1 expression in AfricanAmerican and European-American men, but this may be attributed to small sample size.

Furthermore, precision medicine suggests that genetic polymorphisms and socio-economic factors influence PCa susceptibility and must be taken into account for patient care and treatment of diverse populations. For example, the susceptibility of PCa patients to vitamin D supplementation has been modified by the inheritance of genetic variations in certain populations. ${ }^{49,101,102}$ In a meta-analysis of VDR genetic associations with PCa from 2006 to 2016, genetic variant VDR rs731236 was linked to an elevated risk of PCa development in relation to vitamin D supplementation in Asian-American and African-American men. ${ }^{101}$ Based on 27 case-control studies, VDR gene Fok I polymorphism was linked to higher PCa risk in men with European ancestry. ${ }^{49}$ Also, the VDR rs11568820 variant was related to high risk of aggressive $\mathrm{PCa}$ in vitamin D-deficient African-Americans. ${ }^{49}$ In contrast, VDR genetic alterations in exon 4 and 8 and vitamin D intervention were associated with a protective effect against PCa risk in American men, whereas exons 5, 7, and 9 positively associated with disease susceptibility. ${ }^{103}$ VDR-related variants, HFE (rs9393682) and TUSC3 (rs 1378033), were linked to disease progression in patients with localized tumors (post surgery) and advanced PCA (post androgen deprivation) in two independent cohorts, respectively. ${ }^{62}$ Although both circulating vitamin $E$ plasma levels and intervention have been shown to modulate PCa risk, Vitamin E genetic polymorphisms also influence patient predisposition to develop this malignancy. During vitamin $E$ intervention, inheritance of variants in vitamin E-related genes, SEC14L2, SOD1, and TTPA, significantly modified risk of high-grade PCa in patients. ${ }^{94}$ Two TTPA genetic variants (rs12679996, rs4606052) were linked to elevated mortality risk via inheritance of the CC genotype in high-grade PCa patients. In another study, the NKX3.1 rs11781886 variant (CC,CC + CT) genotypes combined with vitamin $\mathrm{E}$ or selenium treatment increased $\mathrm{PCa}$ risk in a cohort including African-Americans. ${ }^{72}$ Polymorphisms located near BUD13, ZNF259, and APOA5 genes play a role in vitamin $\mathrm{E}$ transport and metabolism and their associated variants modify PCa risk. For example, in a nested case-control study, the BUD13 rs964184 variant was linked to a decrease in PCa risk among men of European ancestry (483 cases and 542 controls). ${ }^{48}$ High lycopene levels in PCa patients (Gleason score $\leq 3+4$ ) were linked to lower Fraction of the Genome Altered. ${ }^{10}$ Inheritance of XRCC1 rs25489 variant and high circulating a-carotene levels in the highest quartile population exerted a protective effect against high-grade PCa in men with the rs25489 GG genotype. High levels of $\beta$-carotene in carriers of the SOD3 rs699473 TC/CC genotype was associated with low risk of high-grade disease. ${ }^{10}$ Selenoprotein-associated polymorphisms were linked to PCa risk among Dana-Farber Cancer Institute patients $(n=722)$ with localized PCa disease. ${ }^{96}$ Dominant genetic models for TXNRD2 rs 1005873 and SELENBP1 rs10788804 were significantly linked to an increase in risk of aggressive PCa. Specifically, TXNRD2 (rs1005873, rs3788310, and rs9606174) variants were linked to higher plasma selenium levels in PCa patients.
Socio-economic status among other social and geographical determinants has been also shown to be associated with elevated PCa incidence and/or mortality in several reports. ${ }^{104-107}$ An inverse relationship exists between socio-economic status and $\mathrm{PCa}$ incidence and mortality. ${ }^{104}$ Cancer screening and detection frequently occur earlier in European-Americans (with high socioeconomic status) compared to African-Americans with the same socio-economic status. Thus, there is a delay between $\mathrm{PCa}$ diagnosis and treatment initiative in African-Americans relative to European-Americans. Moreover, Surveillance, Epidemiology, and End Results (SEER) data showed African-American men had a lower survival rate for surgery and radiation as well as higher incidence of metastasis. Between 2007 and 2011, the SEER registries and US census data showed health insurance had a large effect on disease outcomes for the four leading malignancies in the US. ${ }^{105}$ The most disadvantaged population (median household income $\$ 42,885$ ) were linked to a 1.6-fold increase of distant disease and less likely to receive surgical treatment among patients diagnosed with breast, lung, and PCa relative to the highincome population $(p<0.001)$. These differences were observed across quintiles regardless of the insurance status. ${ }^{105}$ Cancerspecific survival was lowest among most disadvantaged patients. Socio-economic status in the VITAL cohort showed a weak association with PCa risk among men. ${ }^{106}$ However, the susceptibility of cancer-specific mortality was significantly increased among men in age-adjusted, sex-adjusted, and demographicadjusted risk models. African-Americans residing in the Mississippi Delta suffer from higher PCa diagnoses and fatalities compared to average US incidence and mortality rates. ${ }^{107}$ This trend was observed in both rural and urban areas commonly composed of disadvantaged communities. Catchment areas, the surrounding geographic areas and populations that cancer centers service, most often include disadvantaged communities. PCa screening, diagnosis, and treatment access at major cancer centers and hospitals have been shown to differ among certain demographics. The populations serviced within or outside of catchment areas of these cancer centers are associated with lower numbers of African-Americans, Hispanics, and uninsured patients. ${ }^{108,109}$ These patterns may attribute to disadvantaged communities being medically underserved as a result of low socio-economic status or restricted accessibility to medical centers; however, additional studies are needed to validate these relationships.

\section{CONCLUSION AND FUTURE DIRECTIONS}

Recent clinical and population-based efficacy studies have shown the potential of nutraceuticals as potent anti-cancer agents; however, genetic alterations, social determinants, population/ ethnic, and dosage variations modify the protective effect of these agents. Current clinical and epidemiological evidence in diverse populations has not provided sufficient data to determine whether nutraceutical intervention alone or as complement agents can address therapeutic issues associated with health disparities in PCa. Some nutraceutical agents (i.e., Vitamin D, Vitamin $E$, zinc, selenium) have been linked to unintended outcomes in relation to PCa susceptibility due to deficiency, and excessive use of supplements. Therefore, caution should be taken for the therapeutic application of these agents. Furthermore, some epidemiologic evidence implicate differences in PCa susceptibility in diverse populations of men based on genetic variants, socioeconomic, and environmental factors. Specifically, deficient or low levels of Vitamin $D$ and zinc may contribute to health disparities for African-Americans; however, this is still not well understood in the literature. As neo-adjuvant therapies, several nutraceutical have showed limited, but promising inhibitory effects on pathological features associated with precursor neoplastic lesions in PCa. 
Most nutraceutical agents are common constituents in produces or manufactured food products consumed daily by humans and have primarily exhibited non-toxic side effects. Ideally, these agents would serve as potential complement treatment options to overcome toxic side effects exerted by existing clinical therapies against aggressive and advanced PCa in diverse patient populations. Although current clinical and epidemiological evidence identified in this review does not overwhelmingly support the hypothesis of an efficacious and therapeutic role for the selected agents in humans, large efficacy studies for these agents were quite limited and lacked disproportionate populations affected by PCa to demonstrate therapeutic activity in high-risk patients. To fully assess the efficacy of these agents, comprehensive pharmacogenomic, pharmacokinetic, case-control population, and genetic studies and clinical trials are needed to determine lowresponse and high-response rates to and therapeutic limitations of nutraceuticals among diverse patients. Large-scale and inclusive clinical studies will aid to demonstrate whether agents are best utilized as neo-adjuvant or adjuvant therapies to address genetic and pharmacogenomic vulnerabilities in different populations and overcome health disparities. Moreover, these studies will direct the implementation and design of nutraceutical-driven precision medicine strategies to develop patient-focus therapies, reduce the burden of chemotherapy-associated toxicities, suppress disease resistance, and treat both localized and advanced-stage PCa.

\section{ACKNOWLEDGEMENTS}

Original PCa studies in our program are supported by R01 grants CA116636, CA91883, and CA102514.

\section{AUTHOR CONTRIBUTIONS}

D.R. researched, collated the literature, and composed the first draft of the manuscript including Tables 1 and 2. D.R., K.R., and R.A. contributed to the design of the manuscript and significantly edited both the manuscript and Tables 1 and 2 . K. R. and R.A. cross-referenced the literature.

\section{ADDITIONAL INFORMATION}

Competing interests: The authors declare no competing interests.

Publisher's note: Springer Nature remains neutral with regard to jurisdictional claims in published maps and institutional affiliations.

\section{REFERENCES}

1. ACS Cancer Facts and Figures 2018. (American Cancer Society, Atlanta, GA, 2018).

2. Ting, H., Deep, G., Agarwal, C. \& Agarwal, R. The strategies to control prostate cancer by chemoprevention approaches. Mutat. Res. 760, 1-15 (2014).

3. Bijak, M. Silybin, a major bioactive component of milk thistle (Silybum marianum L. Gaernt.)-chemistry, bioavailability, and metabolism. Molecules 22, 1942 (2017).

4. Flaig, T. W. et al. A study of high-dose oral silybin-phytosome followed by prostatectomy in patients with localized prostate cancer. Prostate 70, 848-855 (2010).

5. Nassiri-Asl, M. \& Hosseinzadeh, H. Review of the pharmacological effects of Vitis vinifera (Grape) and its bioactive compounds. Phytother. Res. 23, 1197-1204 (2009).

6. Kaur, M., Agarwal, C. \& Agarwal, R. Anticancer and cancer chemopreventive potential of grape seed extract and other grape-based products. J. Nutr. 139, 1806S-1812S (2009).

7. Brasky, T. M. et al. Specialty supplements and prostate cancer risk in the VITamins and Lifestyle (VITAL) cohort. Nutr. Cancer 63, 573-582 (2011).

8. Capurso, C. \& Vendemiale, G. The mediterranean diet reduces the risk and mortality of theprostate cancer: a narrative review. Front. Nutr. 4, 38 (2017).

9. Key, T. J. et al. Carotenoids, retinol, tocopherols, and prostate cancer risk: pooled analysis of 15 studies. Am. J. Clin. Nutr. 102, 1142-1157 (2015).

10. Nordstrom, T. et al. Associations between circulating carotenoids, genomic instability and the risk of high-grade prostate cancer. Prostate 76, 339-348 (2016).
11. Paur, I. et al. Tomato-based randomized controlled trial in prostate cancer patients: effect on PSA. Clin. Nutr. 36, 672-679 (2017).

12. Wang, Y., Jacobs, E. J., Newton, C. C. \& McCullough, M. L. Lycopene, tomato products and prostate cancer-specific mortality among men diagnosed with nonmetastatic prostate cancer in the Cancer Prevention Study II Nutrition Cohort. Int. J. Cancer 138, 2846-2855 (2016).

13. Graff, R. E. et al. Dietary lycopene intake and risk of prostate cancer defined by ERG protein expression. Am. J. Clin. Nutr. 103, 851-860 (2016).

14. Antwi, S. O. et al. Plasma carotenoids and tocopherols in relation to prostatespecific antigen (PSA) levels among men with biochemical recurrence of prostate cancer. Cancer Epidemiol. 39, 752-762 (2015).

15. Beydoun, H. A., Shroff, M. R., Mohan, R. \& Beydoun, M. A. Associations of serum vitamin $A$ and carotenoid levels with markers of prostate cancer detection among US men. Cancer Causes Control 22, 1483-1495 (2011).

16. Zhang, Y., Coogan, P., Palmer, J. R., Strom, B. L. \& Rosenberg, L. Vitamin and mineral use and risk of prostate cancer: the case-control surveillance study. Cancer Causes Control 20, 691-698 (2009).

17. Kristal, A. R. et al. Dietary patterns, supplement use, and the risk of symptomatic benign prostatic hyperplasia: results from the prostate cancer prevention trial. Am. J. Epidemiol. 167, 925-934 (2008).

18. Meyer, F. et al. Antioxidant vitamin and mineral supplementation and prostate cancer prevention in the SU.VI.MAX trial. Int. J. Cancer 116, 182-186 (2005).

19. Van Hoang, D., Pham, N. M., Lee, A. H., Tran, D. N. \& Binns, C. W. Dietary carotenoid intakes and prostate cancer risk: a case-control study from Vietnam. Nutrients. https://doi.org/10.3390/nu10010070 (2018).

20. Venkitaraman, R. et al. Serum micronutrient and antioxidant levels at baseline and the natural history of men with localised prostate cancer on active surveillance. Tumour Biol. 31, 97-102 (2010).

21. Beilby, J., Ambrosini, G. L., Rossi, E., de Klerk, N. H. \& Musk, A. W. Serum levels of folate, lycopene, beta-carotene, retinol and vitamin $E$ and prostate cancer risk. Eur. J. Clin. Nutr. 64, 1235-1238 (2010).

22. Lane, J. A. et al. Prostate cancer risk related to foods, food groups, macronutrients and micronutrients derived from the UK Dietary Cohort Consortium food diaries. Eur. J. Clin. Nutr. 71, 274-283 (2017).

23. Cui, K. et al. Chemoprevention of prostate cancer in men with high-grade prostatic intraepithelial neoplasia (HGPIN): a systematic review and adjusted indirect treatment comparison. Oncotarget 8, 36674-36684 (2017).

24. Graff, R. E. et al. Circulating antioxidant levels and risk of prostate cancer by TMPRSS2:ERG. Prostate 77, 647-653 (2017).

25. Park, S. Y. et al. Racial/ethnic differences in lifestyle-related factors and prostate cancer risk: the Multiethnic Cohort Study. Cancer Causes Control 26, 1507-1515 (2015).

26. Virtamo, J. et al. Effects of alpha-tocopherol and beta-carotene supplementation on cancer incidence and mortality: 18-year postintervention follow-up of the Alpha-tocopherol, Beta-carotene Cancer Prevention Study. Int. J. Cancer 135, 178-185 (2014).

27. Morgia, G. et al. Association between selenium and lycopene supplementation and incidence of prostate cancer: results from the post-hoc analysis of the procomb trial. Phytomedicine 34, 1-5 (2017).

28. Gann, P. H. et al. A phase II randomized trial of lycopene-rich tomato extract among men with high-grade prostatic intraepithelial neoplasia. Nutr. Cancer 67, 1104-1112 (2015).

29. Magbanua, M. J. et al. Gene expression and biological pathways in tissue of men with prostate cancer in a randomized clinical trial of lycopene and fish oil supplementation. PLoS ONE 6, e24004 (2011).

30. Mahmoud, A. M., Yang, W. \& Bosland, M. C. Soy isoflavones and prostate cancer: a review of molecular mechanisms. J. Steroid Biochem. Mol. Biol. 140, 116-132 (2014).

31. Ahn-Jarvis, J. H. et al. Isoflavone pharmacokinetics and metabolism after consumption of a standardized soy and soy-almond bread in men with asymptomatic prostate cancer. Cancer Prev. Res. 8, 1045-1054 (2015).

32. Lesinski, G. B. et al. Consumption of soy isoflavone enriched bread in men with prostate cancer is associated with reduced proinflammatory cytokines and immunosuppressive cells. Cancer Prev. Res. 8, 1036-1044 (2015).

33. $\mathrm{Wu}, \mathrm{Y}$. et al. Plasma genistein and risk of prostate cancer in Chinese population. Int. Urol. Nephrol. 47, 965-970 (2015).

34. Zhang, H. Y. et al. Isoflavones and prostate cancer: a review of some critical issues. Chin. Med. J. 129, 341-347 (2016).

35. Swami, S. et al. Inhibition of prostaglandin synthesis and actions by genistein in human prostate cancer cells and by soy isoflavones in prostate cancer patients. Int. J. Cancer 124, 2050-2059 (2009).

36. Hamilton-Reeves, J. M., Rebello, S. A., Thomas, W., Kurzer, M. S. \& Slaton, J. W. Effects of soy protein isolate consumption on prostate cancer biomarkers in men with HGPIN, ASAP, and low-grade prostate cancer. Nutr. Cancer 60, 7-13 (2008). 
37. Sur, S. \& Panda, C. K. Molecular aspects of cancer chemopreventive and therapeutic efficacies of tea and tea polyphenols. Nutrition 43-44, 8-15 (2017).

38. McLarty, J. et al. Tea polyphenols decrease serum levels of prostate-specific antigen, hepatocyte growth factor, and vascular endothelial growth factor in prostate cancer patients and inhibit production of hepatocyte growth factor and vascular endothelial growth factor in vitro. Cancer Prev. Res. 2, 673-682 (2009).

39. Thomas, R., Williams, M., Sharma, H., Chaudry, A. \& Bellamy, P. A double-blind, placebo-controlled randomised trial evaluating the effect of a polyphenol-rich whole food supplement on PSA progression in men with prostate cancer-the U.K. NCRN Pomi-T study. Prostate Cancer Prostatic. Dis. 17, 180-186 (2014).

40. Henning, S. M. et al. Randomized clinical trial of brewed green and black tea in men with prostate cancer prior to prostatectomy. Prostate 75, 550-559 (2015).

41. Nguyen, M. M. et al. Randomized, double-blind, placebo-controlled trial of polyphenon $\mathrm{E}$ in prostate cancer patients before prostatectomy: evaluation of potential chemopreventive activities. Cancer Prev. Res. 5, 290-298 (2012).

42. Henning, S. M. et al. Tea polyphenols and theaflavins are present in prostate tissue of humans and mice after green and black tea consumption. J. Nutr. 136, 1839-1843 (2006)

43. Gontero, P. et al. A randomized double-blind placebo controlled phase I-II study on clinical and molecular effects of dietary supplements in men with precancerous prostatic lesions. Chemoprevention or "chemopromotion"?. Prostate 75, 1177-1186 (2015).

44. Bandera Merchan, B., Morcillo, S., Martin-Nunez, G., Tinahones, F. J. \& MaciasGonzalez, M. The role of vitamin D and VDR in carcinogenesis: through epidemiology and basic sciences. J. Steroid Biochem. Mol. Biol. 167, 203-218 (2017).

45. Meyer, H. E. et al. Long term association between serum 25-hydroxyvitamin D and mortality in a cohort of 4379 men. PLOS ONE 11, e0151441 (2016).

46. Batai, K. et al. Race and BMI modify associations of calcium and vitamin D intake with prostate cancer. BMC Cancer 17, 64 (2017).

47. Nelson, S. M., Batai, K., Ahaghotu, C., Agurs-Collins, T. \& Kittles, R. A. Association between Serum 25-hydroxy-vitamin $D$ and aggressive prostate cancer in African American men. Nutrients. https://doi.org/10.3390/nu9010012 (2016).

48. Mondul, A. M., Weinstein, S. J., Layne, T. M. \& Albanes, D. Vitamin D and cancer risk and mortality: state of the science, gaps, and challenges. Epidemiol. Rev. 39 28-48 (2017).

49. Pandolfi, F., Franza, L., Mandolini, C. \& Conti, P. Immune modulation by vitamin D: special emphasis on its role in prevention and treatment of cancer. Clin. Ther. 39, 884-893 (2017).

50. Brandstedt, J., Almquist, M., Manjer, J. \& Malm, J. Vitamin D, PTH, and calcium in relation to survival following prostate cancer. Cancer Causes Control 27, 669-677 (2016).

51. Jarrard, D. et al. Phase Ila, randomized placebo-controlled trial of single high dose cholecalciferol (vitamin D3) and daily Genistein (G-2535) versus double placebo in men with early stage prostate cancer undergoing prostatectomy. Am. J. Clin. Exp. Urol. 4, 17-27 (2016).

52. Medioni, J. et al. Phase I safety and pharmacodynamic of inecalcitol, a novel VDR agonist with docetaxel in metastatic castration-resistant prostate cancer patients. Clin. Cancer Res. 20, 4471-4477 (2014).

53. Xie, D. D. et al. Low vitamin D status is associated with inflammation in patients with prostate cancer. Oncotarget 8, 22076-22085 (2017).

54. Hardiman, G. et al. Systems analysis of the prostate transcriptome in AfricanAmerican men compared with European-American men. Pharmacogenomics 17, 1129-1143 (2016).

55. Brandstedt, J., Almquist, M., Ulmert, D., Manjer, J. \& Malm, J. Vitamin D, PTH, and calcium and tumor aggressiveness in prostate cancer: a prospective nested case-control study. Cancer Causes Control 27, 69-80 (2016).

56. Shui, I. M. et al. Circulating vitamin D, vitamin D-related genetic variation, and risk of fatal prostate cancer in the National Cancer Institute Breast and Prostate Cancer Cohort Consortium. Cancer 121, 1949-1956 (2015).

57. Stephan, C. et al. Serum vitamin D is not helpful for predicting prostate cancer aggressiveness compared with the prostate health index. J. Urol. 196, 709-714 (2016).

58. Dimitrakopoulou, V. I. et al. Circulating vitamin D concentration and risk of seven cancers: Mendelian randomisation study. Br. Med. J. 359, j4761 (2017).

59. Stanaland, M., Jiroutek, M. R. \& Holland, M. A. Study of the association between serum vitamin D levels and prostate cancer. Mil. Med. 182, e1769-e1774 (2017).

60. Gupta, D., Trukova, K., Popiel, B., Lammersfeld, C. \& Vashi, P. G. The association between pre-treatment serum 25-hydroxyvitamin $D$ and survival in newly diagnosed stage IV prostate cancer. PLOS ONE 10, e0119690 (2015).

61. Tagliabue, E., Raimondi, S. \& Gandini, S. Meta-analysis of vitamin D-binding protein and cancer risk. Cancer Epidemiol. Biomark. Prev. 24, 1758-1765 (2015).

62. Lin, V. C. et al. Vitamin D receptor-binding site variants affect prostate cancer progression. Oncotarget 8, 74119-74128 (2017)
63. Giangreco, A. A. et al. Differential expression and regulation of vitamin D hydroxylases and inflammatory genes in prostate stroma and epithelium by 1,25-dihydroxyvitamin $\mathrm{D}$ in men with prostate cancer and an in vitro model. $J$. Steroid Biochem. Mol. Biol. 148, 156-165 (2015).

64. Kyriakopoulos, C. E. et al. A multicenter phase $1 / 2$ a dose-escalation study of the antioxidant moiety of vitamin E 2,2,5,7,8-pentamethyl-6-chromanol (APC-100) in men with advanced prostate cancer. Invest. New Drugs 34, 225-230 (2016).

65. Lawson, K. A. et al. Multivitamin use and risk of prostate cancer in the National Institutes of Health-AARP Diet and Health Study. J. Natl Cancer Inst. 99, 754-764 (2007).

66. Antwi, S. O. et al. Dietary, supplement, and adipose tissue tocopherol levels in relation to prostate cancer aggressiveness among African and European Americans: the North Carolina-Louisiana Prostate Cancer Project (PCaP). Prostate 75, 1419-1435 (2015)

67. Albanes, D. et al. Plasma tocopherols and risk of prostate cancer in the Selenium and Vitamin E Cancer Prevention Trial (SELECT). Cancer Prev. Res. 7, 886-895 (2014).

68. Kristal, A. R. et al. Baseline selenium status and effects of selenium and vitamin e supplementation on prostate cancer risk. J. Natl Cancer Inst. 106, djt456 (2014).

69. Sarre, S., Maattanen, L., Tammela, T. L., Auvinen, A. \& Murtola, T. J. Postscreening follow-up of the Finnish Prostate Cancer Screening Trial on putative prostate cancer risk factors: vitamin and mineral use, male pattern baldness, pubertal development and non-steroidal anti-inflammatory drug use. Scand. J. Urol. 50, 267-273 (2016)

70. Wang, L. et al. Vitamin $E$ and $C$ supplementation and risk of cancer in men posttrial follow-up in the Physicians' Health Study II randomized trial. Am. J. Clin. Nutr. 100, 915-923 (2014)

71. Major, J. M. et al. Genetic variants reflecting higher vitamin e status in men are associated with reduced risk of prostate cancer. J. Nutr. 144, 729-733 (2014).

72. Martinez, E. E. et al. A functional variant in NKX3.1 associated with prostate cancer risk in the Selenium and Vitamin E Cancer Prevention Trial (SELECT). Cancer Prev. Res. 7, 950-957 (2014).

73. Mondul, A. M. et al. Metabolomic analysis of prostate cancer risk in a prospective cohort: the alpha-tocolpherol, beta-carotene cancer prevention (ATBC) study. Int. J. Cancer 137, 2124-2132 (2015).

74. Bauer, S. R. et al. Antioxidant and vitamin E transport genes and risk of highgrade prostate cancer and prostate cancer recurrence. Prostate 73, 1786-1795 (2013).

75. Sapota, A. et al. The bioavailability of different zinc compounds used as human dietary supplements in rat prostate: a comparative study. Biometals 27, 495-505 (2014).

76. van Wijngaarden, E., Singer, E. A. \& Palapattu, G. S. Prostate-specific antigen levels in relation to cadmium exposure and zinc intake: results from the 20012002 National Health and Nutrition Examination Survey. Prostate 68, 122-128 (2008).

77. Gonzalez, A., Peters, U., Lampe, J. W. \& White, E. Zinc intake from supplements and diet and prostate cancer. Nutr. Cancer 61, 206-215 (2009).

78. Eriksen, K. T. et al. Dietary cadmium intake and risk of prostate cancer: a Danish prospective cohort study. BMC Cancer 15, 177 (2015).

79. Lin, Y. S. et al. Increased risk of cancer mortality associated with cadmium exposures in older Americans with low zinc intake. J. Toxicol. Environ. Health $\mathbf{7 6}$ 1-15 (2013).

80. Mondul, A. M., Weinstein, S. J. \& Albanes, D. Vitamins, metabolomics, and prostate cancer. World J. Urol. 35, 883-893 (2017)

81. Rishi, I. et al. Prostate cancer in African American men is associated with downregulation of zinc transporters. Appl. Immunohistochem. Mol. Morphol. 11, 253-260 (2003).

82. Mihelich, B. L. et al. miR-183-96-182 cluster is overexpressed in prostate tissue and regulates zinc homeostasis in prostate cells. J. Biol. Chem. 286, 44503-44511 (2011).

83. Mandair, D., Rossi, R. E., Pericleous, M., Whyand, T. \& Caplin, M. E. Prostate cancer and the influence of dietary factors and supplements: a systematic review. Nutr. Metab. 11, 30 (2014).

84. Singh, B. P. et al. Status and interrelationship of zinc, copper, iron, calcium and selenium in prostate cancer. Indian J. Clin. Biochem. 31, 50-56 (2016).

85. Lu, J. et al. Cancer chemoprevention research with selenium in the post-SELECT era: promises and challenges. Nutr. Cancer 68, 1-17 (2016).

86. Cui, Z., Liu, D., Liu, C. \& Liu, G. Serum selenium levels and prostate cancer risk: a MOOSE-compliant meta-analysis. Medicine 96, e5944 (2017)

87. Hsueh, Y. M. et al. Levels of plasma selenium and urinary total arsenic interact to affect the risk for prostate cancer. Food Chem. Toxicol. 107, 167-175 (2017).

88. Allen, N. E. et al. Selenium and prostate cancer: analysis of individual participant data from fifteen prospective studies. J. Nat/ Cancer Inst. https://doi.org/10.1093/ jnci/djw153 (2016). 
89. Geybels, M. S. et al. Selenoprotein gene variants, toenail selenium levels, and risk for advanced prostate cancer. J. Natl Cancer Inst. https://doi.org/10.1093/jnci/ dju003 (2014).

90. Kok, D. E. et al. A short-term intervention with selenium affects expression of genes implicated in the epithelial-to-mesenchymal transition in the prostate. Oncotarget 8, 10565-10579 (2017).

91. Sinha, R. et al. Selenium-responsive proteins in the sera of selenium-enriched yeast-supplemented healthy African American and Caucasian men. Cancer Epidemiol. Biomark. Prev. 19, 2332-2340 (2010).

92. Eichholzer, M. et al. Effects of selenium status, dietary glucosinolate intake and serum glutathione S-transferase alpha activity on the risk of benign prostatic hyperplasia. BJU Int. 110, E879-E885 (2012).

93. Morgia, G. et al. Effects of Serenoa repens, selenium and lycopene (Profluss(R)) on chronic inflammation associated with benign prostatic hyperplasia: results of "FLOG" (Flogosis and Profluss in Prostatic and Genital Disease), a multicentre Italian study. Int. Braz. J. Urol. 39, 214-221 (2013).

94. Chan, J. M. et al. Selenium- or vitamin E-related gene variants, interaction with supplementation, and risk of high-grade prostate cancer in SELECT. Cancer Epidemiol. Biomark. Prev. 25, 1050-1058 (2016).

95. Gerstenberger, J. P. et al. Selenoprotein and antioxidant genes and the risk of high-grade prostate cancer and prostate cancer recurrence. Prostate 75, 60-69 (2015).

96. Xie, W. et al. Association of genetic variations of selenoprotein genes, plasma selenium levels, and prostate cancer aggressiveness at diagnosis. Prostate 76, 691-699 (2016).

97. Barrington, W. E. et al. Difference in association of obesity with prostate cancer risk between US African American and non-Hispanic white men in the selenium and vitamin E cancer prevention trial (SELECT). JAMA Oncol. 1, 342-349 (2015).

98. Antwi, S. O. et al. Carotenoid intake and adipose tissue carotenoid levels in relation to prostate cancer aggressiveness among African-American and European-American men in the North Carolina-Louisiana prostate cancer project (PCaP). Prostate 76, 1053-1066 (2016).

99. McDonald, A. C., Bunker, C. H., Raman, J., Richie, J. \& Patrick, A. L. Serum carotenoid and retinol levels in African-Caribbean Tobagonian men with high prostate cancer risk in comparison with African-American men. Br. J. Nutr. 117, 1128-1136 (2017).

100. Richards, Z. et al. Prostatic compensation of the vitamin D axis in African American men. JCl Insight 2, e91054 (2017).

101. Liu, S. et al. Association of VDR polymorphisms (Taq I and Bsm I) with prostate cancer: a new meta-analysis. J. Int. Med. Res. 45, 3-10 (2017).

102. Fei, $X$. et al. Polymorphisms of vitamin $D$ receptor gene Taql susceptibility of prostate cancer: a meta-analysis. Onco. Targets Ther. 9, 1033-1045 (2016).

103. Copeland, R. L. et al. DHPLC elution patterns of VDR PCR products can predict prostate cancer susceptibility in African American men. Cancer Genom. Proteom. 14, 461-467 (2017)

104. Pietro, G. D., Chornokur, G., Kumar, N. B., Davis, C. \& Park, J. Y. Racial differences in the diagnosis and treatment of prostate cancer. Int. Neurourol. J. 20, S112-S119 (2016).

105. Abdelsattar, Z. M., Hendren, S. \& Wong, S. L. The impact of health insurance on cancer care in disadvantaged communities. Cancer 123, 1219-1227 (2017).

106. Hastert, T. A., Beresford, S. A., Sheppard, L. \& White, E. Disparities in cancer incidence and mortality by area-level socioeconomic status: a multilevel analysis. J. Epidemiol. Community Health 69, 168-176 (2015).

107. Zahnd, W. E., Jenkins, W. D. \& Mueller-Luckey, G. S. Cancer mortality in the Mississippi Delta Region: descriptive epidemiology and needed future research and interventions. J. Health Care Poor Under. 28, 315-328 (2017).
108. Su, S. C. et al. Spatial analyses identify the geographic source of patients at a National Cancer Institute Comprehensive Cancer Center. Clin. Cancer Res. 16, 1065-1072 (2010).

109. Wang, A. \& Wheeler, D. C. Catchment area analysis using bayesian regression modeling. Cancer Inform. 14, 71-79 (2015).

110. Hamilton-Reeves, J. M. et al. Short-term soy isoflavone intervention in patients with localized prostate cancer: a randomized, double-blind, placebo-controlled trial. PLoS ONE 8, e68331 (2013).

111. deVere White, R. W. et al. Effects of a high dose, aglycone-rich soy extract on prostate-specific antigen and serum isoflavone concentrations in men with localized prostate cancer. Nutr. Cancer 62, 1036-1043 (2010).

112. Lazarevic, B. et al. Efficacy and safety of short-term genistein intervention in patients with localized prostate cancer prior to radical prostatectomy: a randomized, placebo-controlled, double-blind Phase 2 clinical trial. Nutr. Cancer 63, 889-898 (2011).

113. Ahmad, I. U. et al. Soy isoflavones in conjunction with radiation therapy in patients with prostate cancer. Nutr. Cancer 62, 996-1000 (2010).

114. Napora, J. K. et al. High-dose isoflavones do not improve metabolic and inflammatory parameters in androgen-deprived men with prostate cancer. J. Androl. 32, 40-48 (2011).

115. Kwan, W., Duncan, G., Van Patten, C., Liu, M. \& Lim, J. A phase II trial of a soy beverage for subjects without clinical disease with rising prostate-specific antigen after radical radiation for prostate cancer. Nutr. Cancer 62, 198-207 (2010).

116. Ide, H. et al. Combined inhibitory effects of soy isoflavones and curcumin on the production of prostate-specific antigen. Prostate 70, 1127-1133 (2010).

117. Scher, H. I. et al. Randomized, open-label phase III trial of docetaxel plus highdose calcitriol versus docetaxel plus prednisone for patients with castrationresistant prostate cancer. J. Clin. Oncol. 29, 2191-2198 (2011).

118. Kristal, A. R. et al. Plasma vitamin D and prostate cancer risk: results from the selenium and vitamin E cancer prevention trial. Cancer Epidemiol. Biomark. Prev. 23, 1494-1504 (2014).

119. Mondul, A. M. et al. Serum metabolomic response to long-term supplementation with all-rac-alpha-tocopheryl acetate in a randomized controlled trial. J. Nutr. Metab. 2016, 6158436 (2016).

120. Marshall, J. R. et al. Phase III trial of selenium to prevent prostate cancer in men with high-grade prostatic intraepithelial neoplasia: SWOG S9917. Cancer Prev. Res. 4, 1761-1769 (2011)

Open Access This article is licensed under a Creative Commons Attribution 4.0 International License, which permits use, sharing, adaptation, distribution and reproduction in any medium or format, as long as you give appropriate credit to the original author(s) and the source, provide a link to the Creative Commons license, and indicate if changes were made. The images or other third party material in this article are included in the article's Creative Commons license, unless indicated otherwise in a credit line to the material. If material is not included in the article's Creative Commons license and your intended use is not permitted by statutory regulation or exceeds the permitted use, you will need to obtain permission directly from the copyright holder. To view a copy of this license, visit http://creativecommons. org/licenses/by/4.0/.

(c) The Author(s) 2018 